\title{
USP49 deubiquitinates histone H2B and regulates cotranscriptional pre-mRNA splicing
}

\author{
Zhuo Zhang, ${ }^{1,6}$ Amanda Jones, ${ }^{1,6}$ Heui-Yun Joo, ${ }^{1}$ Dewang Zhou, ${ }^{1}$ Ying Cao, ${ }^{2}$ Shaoxia Chen, ${ }^{3}$ \\ Hediye Erdjument-Bromage, ${ }^{4}$ Matthew Renfrow, ${ }^{1}$ Hang He, ${ }^{3}$ Paul Tempst, ${ }^{4}$ Tim M. Townes, ${ }^{1}$ \\ Keith E. Giles, ${ }^{1}$ Ligeng Ma, ${ }^{5}$ and Hengbin Wang ${ }^{1,7^{*}}$ \\ ${ }^{1}$ Department of Biochemistry and Molecular Genetics, University of Alabama at Birmingham, Birmingham, Alabama 35294, \\ USA; ${ }^{2}$ State Key Laboratory of Cellular and Molecular Biology, College of Life Science, Hebei Normal University, Shijiazhuang, \\ Hebei 050021, China; ${ }^{3}$ College of Life Sciences, Peking University, Beijing 100871, China; ${ }^{4}$ Molecular Biology Program, Memorial \\ Sloan Kettering Cancer Center, New York, New York 10021, USA; ${ }^{5}$ College of Life Sciences, Capital Normal University, Beijing \\ 100048, China
}

Post-translational histone modifications play important roles in regulating chromatin structure and function. Histone H2B ubiquitination and deubiquitination have been implicated in transcriptional regulation, but the function of $\mathrm{H} 2 \mathrm{~B}$ deubiquitination is not well defined, particularly in higher eukaryotes. Here we report the purification of ubiquitin-specific peptidase 49 (USP49) as a histone H2B-specific deubiquitinase and demonstrate that H2B deubiquitination by USP49 is required for efficient cotranscriptional splicing of a large set of exons. USP49 forms a complex with RuvB-like1 (RVB1) and SUG1 and specifically deubiquitinates histone H2B in vitro and in vivo. USP49 knockdown results in small changes in gene expression but affects the abundance of $>9000$ isoforms. Exons down-regulated in USP49 knockdown cells show both elevated levels of alternative splicing and a general decrease in splicing efficiency. Importantly, USP49 is relatively enriched at this set of exons. USP49 knockdown increased H2B ubiquitination (uH2B) levels at these exons as well as upstream $3^{\prime}$ and downstream 5' intronic splicing elements. Change in H2B ubiquitination level, as modulated by USP49, regulates U1A and U2B association with chromatin and binding to nascent pre-mRNA. Although $\mathrm{H} 3$ levels are relatively stable after USP49 depletion, H2B levels at these exons are dramatically increased, suggesting that uH2B may enhance nucleosome stability. Therefore, this study identifies USP49 as a histone H2B-specific deubiquitinase and uncovers a critical role for $\mathrm{H} 2 \mathrm{~B}$ deubiquitination in cotranscriptional pre-mRNA processing events.

[Keywords: USP49; H2B deubiquitination; transcription; pre-mRNA splicing]

Supplemental material is available for this article.

Received November 27, 2012; revised version accepted June 12, 2013.

Post-translational modification of the short C-terminal and long $\mathrm{N}$-terminal histone tails has emerged as a key modulator of chromatin structure and function (Jenuwein and Allis 2001; Kouzarides 2007). Histone ubiquitination, which occurs primarily on histone $\mathrm{H} 2 \mathrm{~A}$ and $\mathrm{H} 2 \mathrm{~B} \mathrm{C}$ termini, is a unique modification involving the addition of a bulky ubiquitin moiety, which influences chromatin structure directly and/or serves as a platform for the exclusion of effector proteins (Wyce et al. 2007; Fleming et al. 2008; Chandrasekharan et al. 2009). Unlike H2A ubiquitination ( $\mathrm{uH} 2 \mathrm{~A})$, the evolutionarily conserved $\mathrm{uH} 2 \mathrm{~B}$ is enriched at

\footnotetext{
${ }^{6}$ These authors contributed equally to this work.

${ }^{7}$ Corresponding author

E-mail hbwang@uab.edu

Article published online ahead of print. Article and publication date are online at http://www.genesdev.org/cgi/doi/10.1101/gad.211037.112.
}

transcribed regions of the genome, and $\mathrm{uH} 2 \mathrm{~B}$ abundance generally correlates with gene expression level (Minsky et al. 2008; Batta et al. 2011; Schulze et al. 2011). Indeed, $\mathrm{H} 2 \mathrm{~B}$ ubiquitination depends on active transcription and requires activator-dependent recruitment of the Rad6/ Bre1 H2B ubiquitination machinery and the elongating RNA polymerase II (RNAP II)-associated PAF complex (Weake and Workman 2008). During transcription, RNAP II acts as a scaffold for the assembly of chromatin-modifying/ remodeling complexes and pre-mRNA processing factors, facilitating transcription and cotranscriptional pre-mRNA processing (Shukla and Oberdoerffer 2012). Emerging evidence indicates that chromatin remodeling and histone modifications may influence pre-mRNA processing by regulating RNAP II elongation rate and splicing factor recruitment (Allo et al. 2009; Schor et al. 2009; Carrillo Oesterreich et al. 2011). uH2B is enriched at intron-exon 
junctions in budding yeast and mammalian cells (Shieh et al. 2011; Jung et al. 2012), and uH2B was recently shown to interact with Npl3, a yeast serine-arginine-rich (SR)-like protein required for recruitment of core splicing factors (Moehle et al. 2012). However, a direct role for $\mathrm{uH} 2 \mathrm{~B}$ in pre-mRNA processing events has not been demonstrated in mammals.

Ubiquitination of histone $\mathrm{H} 2 \mathrm{~B}$ is a dynamic process. Two H2B deubiquitinases-Ubp8 and Ubp10 - deubiquitinate $\mathrm{H} 2 \mathrm{~B}$ in budding yeast, with Ubp8 specifically implicated in transcription regulation (Henry et al. 2003; Daniel et al. 2004; Emre et al. 2005; Gardner et al. 2005). Drosophila contains three putative H2B deubiquitinases: the Ubp8 and Ubp10 homologs Nonstop and Scrawny and the nonhomologous ubiquitin-specific peptidase 7 (USP7) protein (van der Knaap et al. 2005; Weake et al. 2008; Buszczak et al. 2009). The number of H2B deubiquitinases is further expanded in mammals. USP3, USP12, USP22 (Ubp8 homolog), USP44, and USP46 are reported to have H2B deubiquitination activity in mammals (Nicassio et al. 2007; Zhang et al. 2008; Zhao et al. 2008; Joo et al. 2011; Fuchs et al. 2012). These H2B deubiquitinases may function in distinct chromatin domains, developmental stages, and cell types. However, uH2A may also be a substrate for these deubiquitinases, complicating the interpretation of their in vivo functions. It is currently not known whether mammalian genomes contain H2B-specific deubiquitinases. The evolutionarily driven expansion of the USP family of deubiquitinases suggests that there may be as yet undefined $\mathrm{H} 2 \mathrm{~B}$ deubiquitinases.

In light of these unanswered questions, we sought to identify histone $\mathrm{H} 2 \mathrm{~B}$ deubiquitinases in human cells. Using biochemical purification techniques, we identified USP49 as a H2B-specific deubiquitinase. Functional characterization revealed that USP49 specifically regulates $\mathrm{uH} 2 \mathrm{~B}$ levels, and USP49 knockdown is associated with genome-wide changes in cotranscriptional splicing. Therefore, this study identifies USP49 as a novel H2B deubiquitinase and reveals a previously unknown function for $\mathrm{H} 2 \mathrm{~B}$ deubiquitination in pre-mRNA processing.

\section{Results}

Purification of USP49 as a putative histone H2B deubiquitinase

To identify putative $\mathrm{H} 2 \mathrm{~B}$ deubiquitinases, we assayed the deubiquitinase activity of HeLa cell nuclear proteins using uH2B-containing nucleosome and histone substrates (Joo et al. 2011). As shown in Figure 1A, nuclear extract (NE) P11 0.3 M, 0.5 M, and 1.0 M fractions and nuclear pellet (NP) DE52Ft and P11 0.3 M, 0.5 M, and 1.0 $\mathrm{M}$ fractions exhibit robust $\mathrm{H} 2 \mathrm{~B}$ deubiquitination activities toward nucleosome substrates (Fig. 1A, bottom panel, cf. lanes 3-6,8-10 and 1). We focused on the P11 NE0.5 fraction, as this fraction contains robust, nucleosome-specific deubiquitination activity (Fig. 1A, cf. top and bottom panels). The NE P11 0.5 M H2B deubiquitination activity split into three peaks following DEAE5PW fractionation (Fig. 1C). While purification of the first two peaks is ongoing, we purified the third peak through four additional columns (Fig. 1B). In the final step, a Superose 6 column separation, the H2B deubiquitination activity correlated with several polypeptides on SDS-PAGE (Fig. 1D, top two panels, candidate bands are marked with red asterisks in the silver-stained panel). Due to the limited amount of sample, we employed mass spectrometry to identify all polypeptides in these fractions. Mass spectrometry analysis of the polypeptides in fraction \#60 identified USP49, a zinc finger-containing C19 peptidase family member (Fig. 1D, top panel, candidate band is marked with a red arrow in the silver-stained gel, and identified peptides are labeled on the right side of the gel), which shares significant sequence similarity and domain structure with other reported histone deubiquitinases (Fig. 2A). Western blot analyses of fractions from the DEAE-5PW, Superose 6, and other columns revealed that USP49 consistently coelutes with $\mathrm{uH} 2 \mathrm{~B}$ deubiquitination activity during purification (Fig. 1C [bottom panel], D [bottom panels]; data not shown), suggesting that USP49 is a histone $\mathrm{H} 2 \mathrm{~B}$ deubiquitinase.

\section{USP49 interacts with RuvB-like1 (RVB1) and SUG1 and specifically deubiquitinates $H 2 B$ in vitro}

To confirm that USP49 has $\mathrm{uH} 2 \mathrm{~B}$ deubiquitination activity, we purified recombinant USP49 from sf9 cells to homogeneity by sequential anti-Flag and anti-Myc affinity purification and tested the protein for deubiquitination activity (Fig. 2B, left panel). Baculovirus-expressed USP49 failed to efficiently deubiquitinate $\mathrm{uH} 2 \mathrm{~B}$ in either core histone or nucleosome form (Fig. 2C, middle and bottom panels, cf. lanes 1,2 and 5,6). Surprisingly, USP49 deubiquitinates core histone but not nucleosomal uH2A (Fig. 2D, second and fourth panels, cf. lanes 1,2 and 5,6). This result suggested that USP49 may require interacting proteins or specific regulatory post-translational modifications for $\mathrm{uH} 2 \mathrm{~B}$-specific deubiquitination activity. Since the mass spectrometry protocol used to identify USP49 was incompatible with the identification of post-translational modifications, we purified the USP49 complex from a HeLa S3 cell line stably expressing Flag-HA USP49 (Fig. 2B, middle panel). Mass spectrometry analysis of USP49 immunoprecipitates identified RVB1 (Fig. 2B, middle panel, 52-kDa band; Supplemental Fig. S1) and 26S proteasome regulatory subunit 5 (SUG1 or PMSC5) (Fig. 2B, middle panel, 45-kDa band; Supplemental Fig. S1) in addition to USP49 (Fig. 2B, middle panel, 72-kDa band; Supplemental Fig. S1). RVB1, an ATPase and putative helicase, is an integral subunit of many chromatin remodeling/modification complexes and is proposed to mediate interactions between nucleosomes and these chromatin remodelers (Jha and Dutta 2009). SUG1 is an ATPase and a subunit of the 19S regulatory particle. This protein has been shown to mediate the regulation of H3K4 and H3K79 methylation by $\mathrm{uH} 2 \mathrm{~B}$ in budding yeast (Ezhkova and Tansey 2004). Western blot assay confirmed that the anti-USP49 antibody, but not control IgG, specifically immunoprecipitates RVB1 and SUG1 from crude NEs (Supplemental Fig. S2, cf. lanes 2 
A

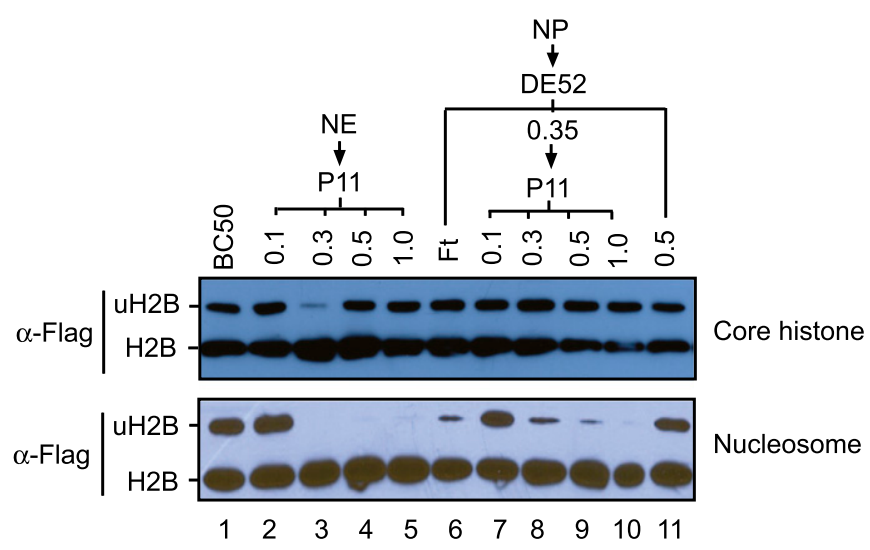

B

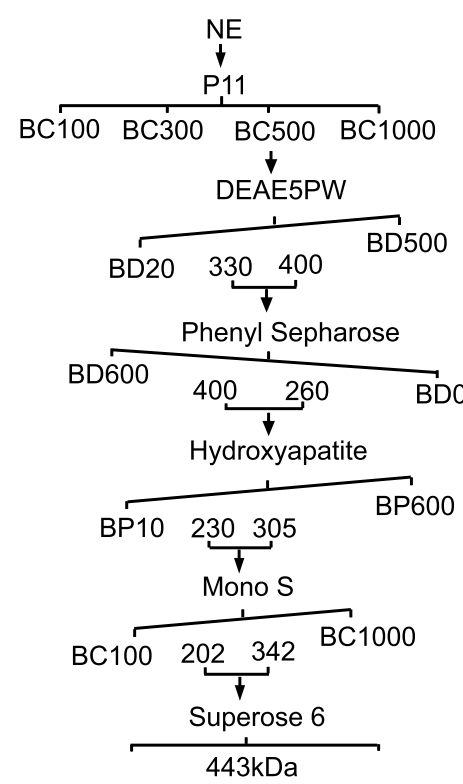

C

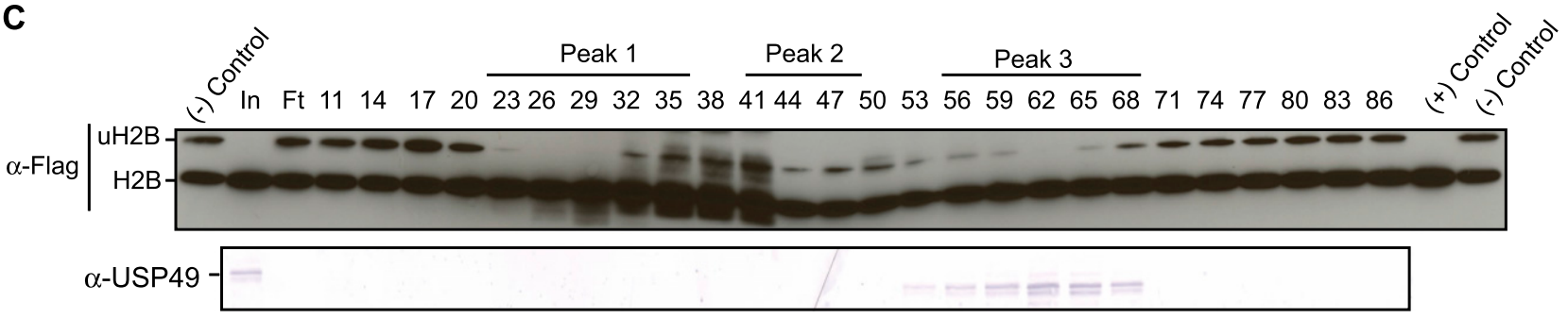

D

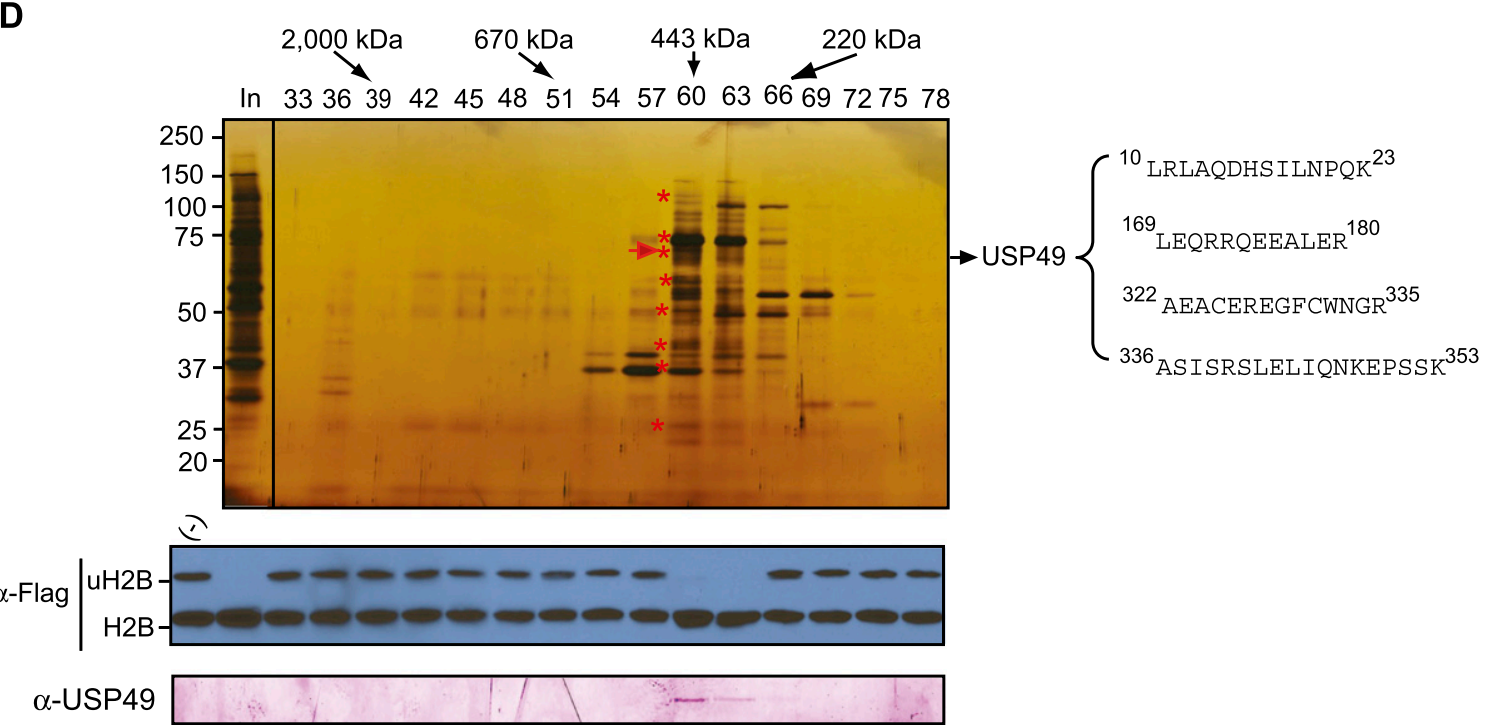

Figure 1. Purification of USP49 as a putative histone H2B deubiquitinase. (A) H2B deubiquitination assay with HeLa cell nuclear proteins fractionated on DE52 and P11 columns. Numbers indicate the salt concentration (molar) for step elution. The top and bottom panels use core histone and nucleosome as substrates, respectively. $(B)$ Schematic representation of the steps used to purify the histone H2B deubiquitinase. Numbers represent the salt concentrations (millimolar) at which the H2B deubiquitinase activity elutes from the columns. $(C)$ H2B deubiquitination assay (top panel) and Western blot analysis (bottom panel) of the fractions derived from the DEAE5PW column. Antibodies are indicated at the left side of the panel. NE 0.5 M was used as positive control. BC50 was used as negative control. $(D)$ Silver staining of a polyacrylamide-SDS gel (top panel), H2B deubiquitination activity assay (second panel), and Western blot analysis (bottom panel) of the fractions derived from the Superose 6 column. Candidate bands are labeled with red asterisks. USP49 is also indicated by an arrow, and identified peptides are labeled on the right side of the top panel. The elution profile of the protein markers is indicated at the top of the panel. 
A

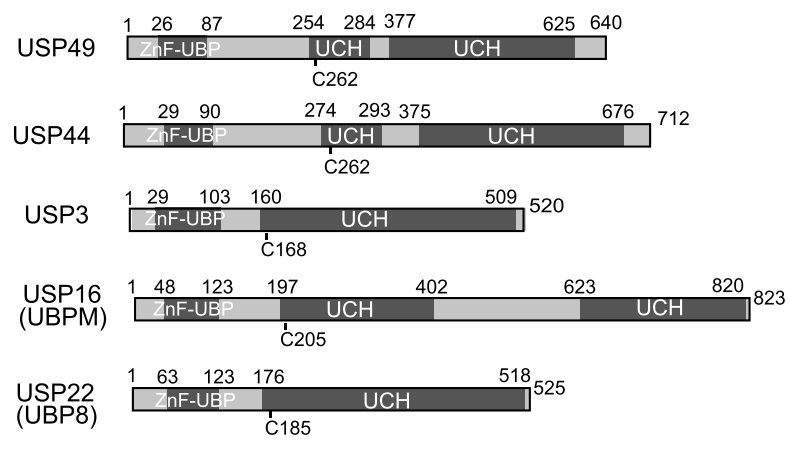

C

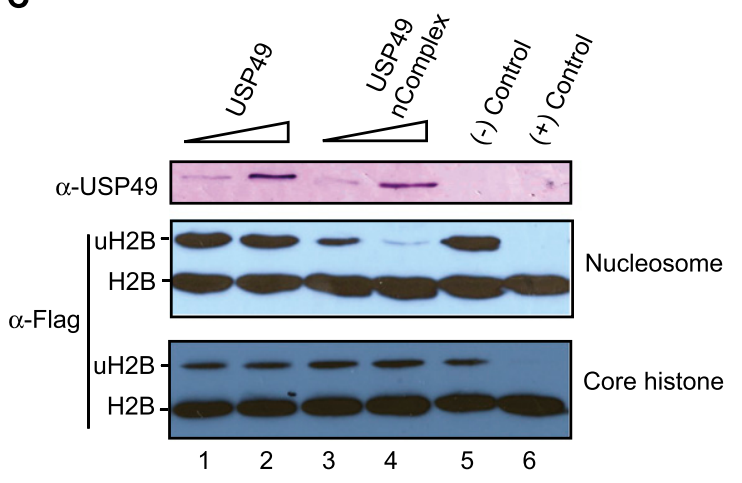

E

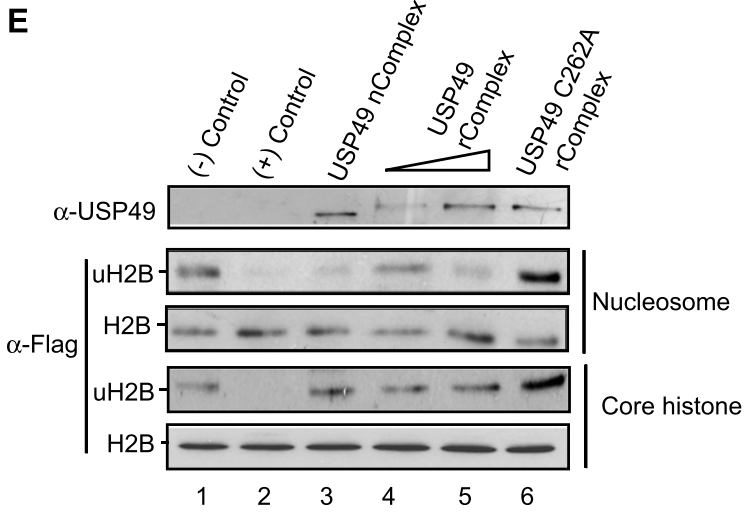

B

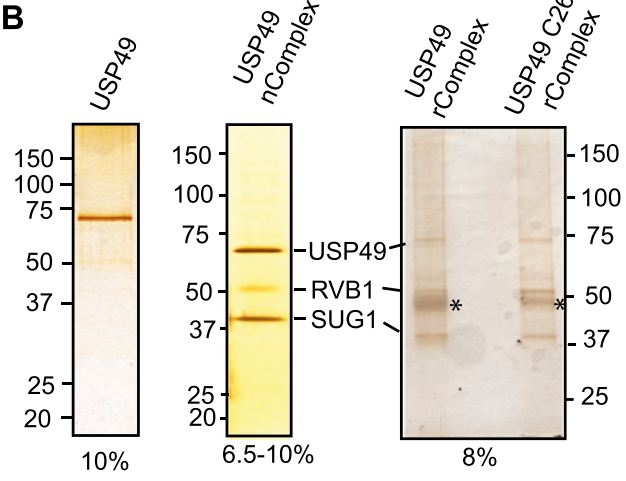

D

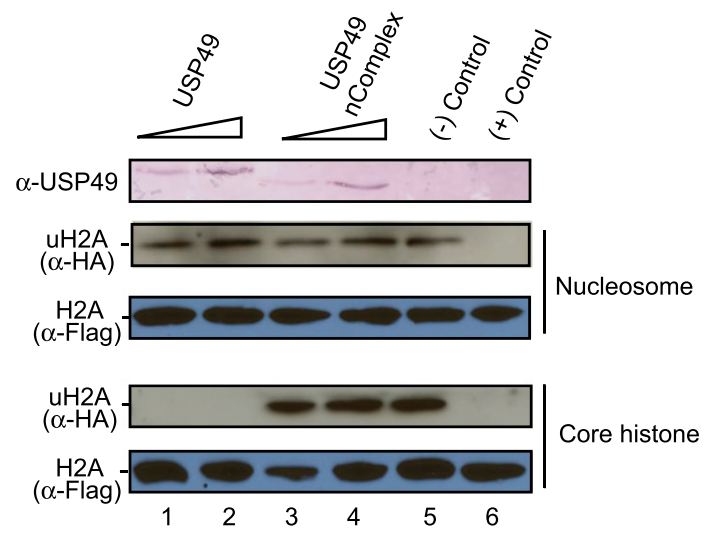

F

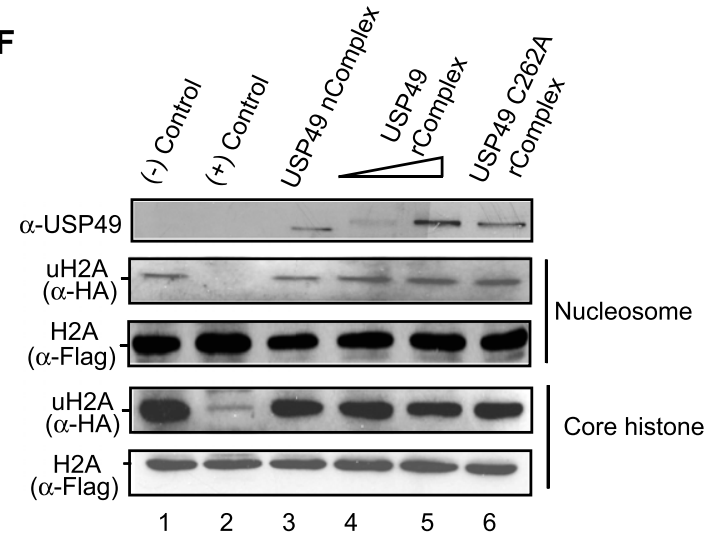

Figure 2. USP49 interacts with RVB1 and SUG1 and specifically deubiquitinates uH2B in vitro. (A) Schematic representation of USP49, USP44, USP3, USP16 (Ubp-M), and USP22 (Ubp-8). Positions of ZnF-UBP and UCH domains are shown. The cysteine residues corresponding to the catalytic triad are also shown. Numbers represent the amino acid position. (B) Silver staining of a SDS-PAGE containing USP49 purified from sf9 cells (left), the USP49 complex from the HeLa S3 stable cell line (middle), and the reconstituted wildtype USP49 and C262A mutant USP49 complexes (right). The identities of each polypeptide are labeled. Peptides identified by mass spectrometry analysis are included in Supplemental Figure S1. (C) H2B deubiquitination assay of USP49 and the USP49 complex. The top panel shows the amount of USP49 used in the assay. Note: USP49 purified from sf9 cells migrated slower than USP49 purified from HeLa stable cell lines on SDS-PAGE because two Myc tags were added to the USP49 C terminus. The middle panel shows assays with nucleosomes as substrates, and the bottom panel shows assays with core histone as substrates. The NE $0.5 \mathrm{M}$ fraction was used as a positive control for nucleosomes, and the NE $0.3 \mathrm{M}$ fraction was used as a positive control for core histone. BC50 was used as a negative control. $(D)$ H2A deubiquitination assay of USP49 and the USP49 complex. The top panel shows the amount of USP49 used in the assay. The second and third panels show assays with nucleosomes as substrates, and the bottom two panels show assays with core histone as substrates. The NE $0.5 \mathrm{M}$ fraction was used as a positive control. BC50 was used as a negative control. $(E) \mathrm{H} 2 \mathrm{~B}$ deubiquitination assay of reconstituted wild-type and mutant USP49 complex and the USP49 complex purified from HeLa cells. The top panel shows the amount of USP49 used in the assay. The middle panel shows assays with nucleosomes as substrates, and the bottom panel shows assays with core histone as substrates. The NE $0.5 \mathrm{M}$ fraction was used as a positive control for nucleosomes, and the NE $0.3 \mathrm{M}$ fraction was used as a positive control for core histone. BC50 was used as a negative control. $(F)$ H2A deubiquitination assay of reconstituted wild-type and mutant USP49 complex and the USP49 complex purified from HeLa cells. The top panel shows the amount of USP49 used in the assay. The second and third panels show assays with nucleosomes as substrates, and the bottom two panels show assays with core histone as substrates. The NE 0.5 M fraction was used as a positive control. BC50 was used as a negative control. 
and 3). To determine the substrate specificity of the USP49 complex, we performed in vitro deubiquitination assays with $\mathrm{uH} 2 \mathrm{~B}$ - or uH2A-containing histone or mononucleosome substrates. We found that USP49, in complex with RVB1 and SUG1, can efficiently deubiquitinate uH2B-containing mononucleosomes (Fig. 2C, middle panel, cf. lanes 3,4 and 5,6 [note that recombinant USP49 is triple-tagged, accounting for the slower migration]). However, the USP49 complex displays no activity toward histone $\mathrm{uH} 2 \mathrm{~B}$, histone $\mathrm{uH} 2 \mathrm{~A}$, or nucleosomal $\mathrm{uH} 2 \mathrm{~A}$ (Fig. 2C [bottom panel, cf. lanes 3,4 and 5,6], D [second and fourth panels, cf. lanes 3,4 and 5,6]). These results suggest that RVB1 and SUG1 may regulate USP49's enzymatic activity and/or substrate specificity. To confirm that USP49 indeed forms a complex with SUG1 and RVB1 and deubiquitinates histone $\mathrm{H} 2 \mathrm{~B}$, we reconstituted the USP49-RVB1-SUG1 complex in sf9 cells. Both wildtype and catalytically inactive C262A mutant USP49 can form complexes with SUG1 and RVB1 (Fig. 2B, right panel). Importantly, complex reconstituted with wildtype USP49 exhibits H2B deubiquitination activity and substrate specificity similar to the USP49 complex purified from HeLa cells (Fig. 2E,F, second and fourth panels, cf. lanes 4,5 and 3). In contrast, complex reconstituted with USP49 C262A mutant does not have deubiquitination activities toward $\mathrm{H} 2 \mathrm{~B}$ and $\mathrm{H} 2 \mathrm{~A}$ in histone or nucleosome form (Fig. 2E,F, cf. lanes 6 and 3-5). This result confirms USP49 as the catalytic subunit and RVB1 and SUG1 as regulatory subunits of the USP49 complex.

\section{USP49 mediates H2B deubiquitination in vivo}

To determine whether USP49 deubiquitinates uH2B and/ or $\mathrm{uH} 2 \mathrm{~A}$ in vivo, we first investigated whether overexpression of USP49 affects histone ubiquitination. For this purpose, we transfected expression vectors encoding wild-type USP49 or the catalytically inactive USP49 C262A mutant into 293T cells (Fig. 3A). Overexpression of wild-type but not C262A mutant USP49 resulted in a significant reduction of $\mathrm{uH} 2 \mathrm{~B}$ levels (Fig. 3A, third panel, cf. lanes 2,3 and 1 ). The reduction in histone ubiquitination is specific for $\mathrm{uH} 2 \mathrm{~B}$, as no change in $\mathrm{H} 2 \mathrm{~A}$ ubiquitination levels was observed in cells expressing either wild-type or mutant USP49 (Fig. 3A, fourth panel, cf. lanes 2,3 and 1). To further determine the function of USP49 in uH2B deubiquitination, we examined whether knockdown of USP49 affects H2B ubiquitination levels. As shown in Figure 3B, transfection of siRNA against USP49 generated a dose-dependent decrease in USP49 protein levels (Fig. 3B, top two panels, cf. lanes 2,3 and 1). Importantly, levels of $\mathrm{uH} 2 \mathrm{~B}$ were also increased in a dosedependent manner in these cells (Fig. 3B, third panel, cf. lanes 2,3 and 1), while uH2A levels were unaffected (Fig. 3B, fourth panel, cf. lanes 2,3 and 1). To ensure that the increase in $\mathrm{uH} 2 \mathrm{~B}$ did not result from potential offtarget effects of siRNA, we established inducible USP49 knockdown HCT116 cell lines using different targeting sequences. Doxycycline-induced USP49 knockdown led to a dramatic reduction in USP49 protein levels (Supplemental Fig. S3, top panel, USP49 is marked with arrow,
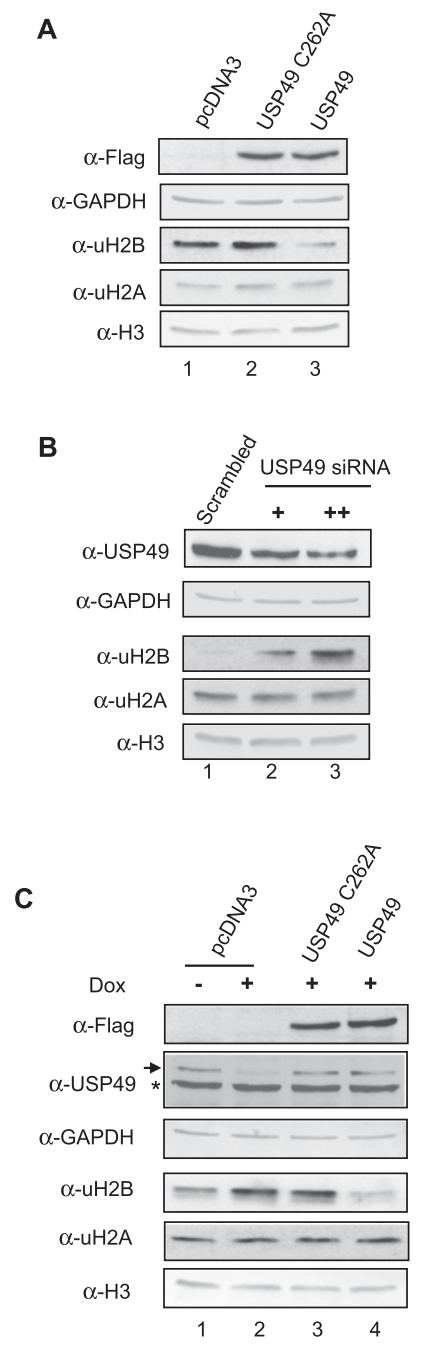

Figure 3. USP49 deubiquitinates $\mathrm{uH} 2 \mathrm{~B}$ in vivo. $(A)$ Overexpression of wild-type but not C262A mutant USP49 reduces the levels of uH2B in $293 \mathrm{~T}$ cells. Western blot assay of cells transfected with expression vectors as indicated at the top of the panels. Antibodies used are indicated at the left side of the panels. (B) Knockdown of USP49 results in an increase of the levels of uH2B in 293T cells. Cells were transfected with siRNA as indicated at the top of the panels and were subjected to Western blot assay. Antibodies used are indicated at the left side of the panels. (C) Expression of wild-type but not C262A mutant USP49 restores histone uH2B levels induced by USP49 knockdown. USP49-inducible knockdown cells were transfected with wild-type and C262A mutant USP49 and subjected to Western blot assay. USP49 is marked with an arrow, and nonspecific bands are marked with asterisks. Antibodies used are indicated at the left side of the panels.

and nonspecific bands are marked with asterisks) accompanied by a specific increase of $\mathrm{H} 2 \mathrm{~B}$ ubiquitination levels (Supplemental Fig. S3, cf. third and fourth panels, cf. lanes 1,3 and 2,4). Importantly, the effect of USP49 knockdown on $\mathrm{H} 2 \mathrm{~B}$ deubiquitination could be specifically rescued by expression of wild-type but not C262A mutant USP49 (Fig. 3C, fourth panel, cf. lanes 3,4 and 1,2). Based on these data, we conclude that the USP49 complex specifically deubiquitinates histone $\mathrm{H} 2 \mathrm{~B}$ in vivo. 
USP49 depletion affects cotranscriptional pre-mRNA splicing

Since USP49 deubiquitinates uH2B, we investigated whether USP49 regulates gene expression by performing RNA sequencing (RNA-seq) experiments. We performed a differential expression analysis and identified $\sim 170$ genes significantly $(P=0.05)$ up-regulated or downregulated in USP49 knockdown cells (Fig. 4A). Median $\log _{2}$ fold change in the expression of these genes was slightly negative, suggesting that USP49 may act as a positive regulator of transcription (Fig. 4A). Interestingly, we found that USP49 significantly regulated the differential expression of $>9000$ gene isoforms, suggesting that, in addition to functions in transcriptional regulation, USP49 may also regulate pre-mRNA splicing (Fig. 4A). To determine whether USP49 affects splicing, we counted the number of spliced tags indicative of exon inclusion and exon skipping for each internal exon. Next, we calculated the percentage of inclusive splicing events for each internal exon in control and USP49 knockdown cells (see the Materials and Methods). Intriguingly, we found that exons down-regulated in USP49 knockdown cells also showed a small but statistically significant decrease in the rate of exon inclusion (Fig. 4B). Upon visual inspection of these exons, we observed a consistent relative increase in the number of tags aligning to flanking introns after USP49 knockdown, suggesting that USP49 may regulate the efficiency of splicing for these exons (Fig. 4C). Since alternative exons are often more slowly spliced in vivo (Ameur et al. 2011; Tilgner et al. 2012), we determined whether USP49 knockdown affects splicing efficiency by calculating a measure of splicing completion (SC) for each internal exon (see the Materials and Methods). As expected, we found that most exons are completely spliced (SC > 0.95) (Supplemental Fig. S4), although exons down-regulated in USP49 knockdown cells have generally lower SC values both before and after USP49 knockdown (Supplemental Fig. S5). Consistent with our results demonstrating that exons down-regulated in USP49 knockdown cells show higher levels of exon exclusion, we found that these exons also show a decrease in median SC value after USP49 knockdown (Supplemental Fig. S6). To quantify the relative increase in unspliced transcripts in USP49 knockdown cells, we calculated the fold change in unspliced transcripts for all internal exons. We found that exons down-regulated in USP49 knockdown cells show a slightly positive fold change in unspliced transcripts, with a maximal change of nearly threefold (Fig. 4D). To confirm that these changes were not an artifact of RNA-seq normalization, we performed oligo-dt-primed RT-qPCR assays for exons with lower SC values in USP49 knockdown cells. As shown in Figure 4E, RPS3 and RPS6 intron-containing transcripts are enriched greater than fourfold in USP49 knockdown cells. Recent work has suggested that splicing is the final licensing factor for release of polyadenylated transcripts from chromatin (Gu et al. 2013). To determine the subcellular localization of aberrant transcripts in USP49 knockdown cells, we purified chroma- tin-bound and nucleoplasmic mRNA (Tilgner et al. 2012). The efficiency of cotranscriptional splicing is severely impaired in USP49 knockdown cells, as evidenced by the increase in intron-containing transcripts in the chromatin-bound fraction (Fig. 4F,G; Supplemental Fig. S7A-C, red bars). To determine whether the elevated levels of partially spliced mRNA in USP49 knockdown cells are the result of defects in splicing or defects in mRNA surveillance, we monitored mRNA decay rate in control and knockdown cells. We performed RT-qPCR analysis on total mRNA isolated from control and knockdown cells at different time points after actinomycin D inhibition of transcription. As shown in Supplemental Figure S8A-D, intron levels are elevated in USP49 knockdown cells, confirming our RT-qPCR and RNA-seq results. Interestingly, the half-life of intron-containing mRNA is not affected by USP49 knockdown $(\sim 1 \mathrm{~h})$. Intron-containing transcripts are rapidly degraded and return to control levels within $\sim 2 \mathrm{~h}$ (Supplemental Fig. S8A-D). These results suggest that USP49 affects premRNA splicing but not mRNA decay.

To more precisely define the relationship between USP49 and splicing, we performed anti-Flag chromatin immunoprecipitation (ChIP) in a USP49-inducible knockdown cell line stably expressing Flag-USP49. We found that USP49 is highly enriched near the transcription start site and is relatively enriched at coding regions of the genome (Fig. 6A, below). More than 4000 genes are bound by USP49 (MACS, mfold > 8), the majority of which are housekeeping genes (data not shown). Interestingly, nearly 3000 of the exons down-regulated in USP49 knockdown cells are transcribed from these genes. This set of exons shows a larger increase in exon skipping and a larger decrease in splicing efficiency in USP49 knockdown cells than the set of all exons down-regulated in USP49 knockdown cells (Supplemental Figs. S9, S10). We therefore focused our analysis on exons from genes bound by USP49. Importantly, although exons from genes bound by USP49 are both up-regulated and down-regulated in USP49 knockdown cells, we found that the downregulated exons are significantly enriched for USP49 binding (Fig. 5A,B). USP49 is also enriched in regions spanning their $5^{\prime}$ and $3^{\prime}$ ( \pm 500 nucleotides [nt]) splice sites (Fig. 5A,B; Supplemental Fig. S11). As shown in Supplemental Figure S12, down-regulated exons from USP49-bound genes show a median increase in unspliced transcripts. To determine whether USP49 mediates these splicing defects, we performed RT-qPCR validation. As shown in Figure 5, C and D, the increase in introncontaining pre-mRNA in USP49 knockdown cells could be rescued by expression of wild-type but not C262A mutant USP49. Similar rescue effects were seen for other genes (data not shown). These data demonstrate that it is the USP49 deubiquitinase activity that regulates mRNA splicing. To determine whether splicing regulation is a common feature of histone $\mathrm{H} 2 \mathrm{~B}$ deubiquitinases or a unique function of USP49, we examined the effect of knockdown of other known histone $\mathrm{H} 2 \mathrm{~B}$ deubiquitinases on mRNA splicing. Knockdown of USP22 and USP12 had no detectable effect on splicing for any of the examined 
A

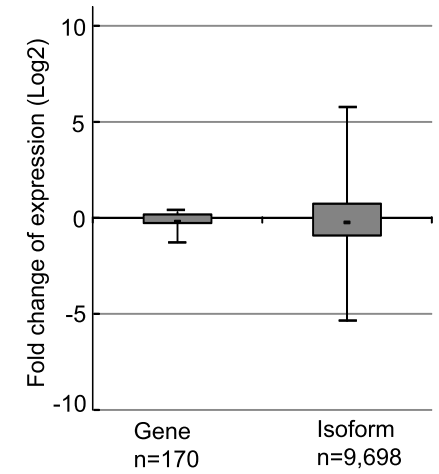

B

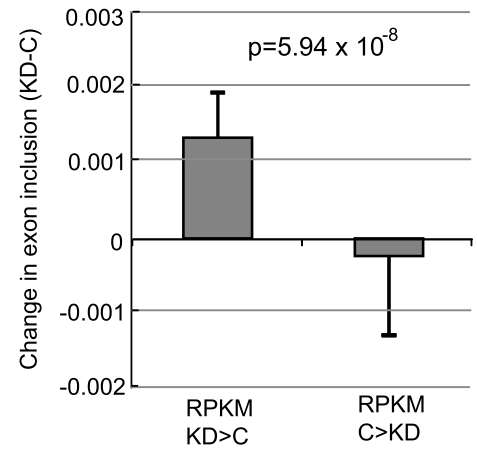

C

1.3 fold increase of unspliced transcipts Scale

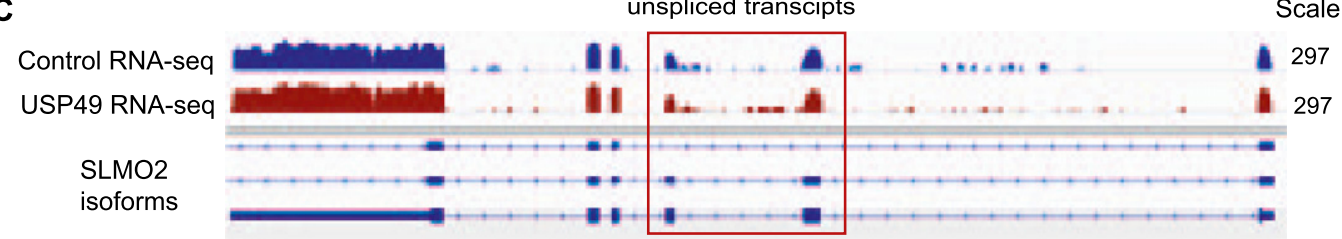

D

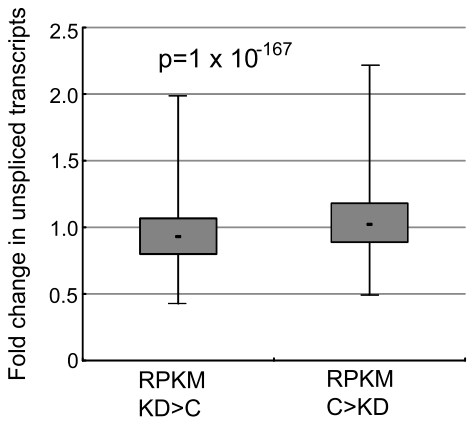

$\mathbf{F}$



$\mathbf{E}$


G

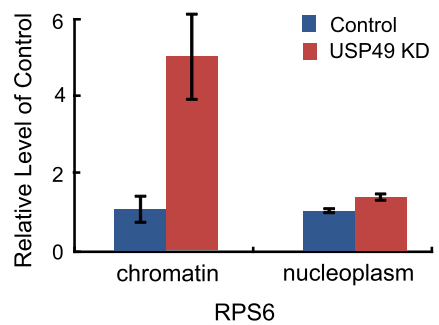

Figure 4. USP49 depletion affects pre-mRNA splicing. (A) A box and whisker plot of fold change $\left(\log _{2}\right)$ in differentially expressed genes and transcripts (cutoff value, $P=0.05$ ) in control and USP49 knockdown cells as determined by RNA-seq analysis. The lower end of the box represents the 25th percentile, the upper end of the box represents the 75th percentile, the bar represents the median, and the upper and lower whiskers represent the 99th and first percentiles, respectively. (B) The mean (columns) and variance (bars) of change in exon inclusion levels for exons up-regulated or down-regulated in USP49 knockdown cells. Statistical significance was calculated using a Student's $t$-test. $(C)$ A representative image of the SLMO2 transcript, showing a relative increase in intron-aligning tags in USP49 knockdown cells. The control RNA-seq is shown in blue, and the USP49 knockdown are in red. The exons (thick blue boxes) and introns (thin blue lines) are shown at the bottom of the panels. The region showing decreased splicing efficiency is indicated by the red box. (D) A box and whisker plot, as described in Figure 3A, showing the fold change in unspliced transcripts for exons up-regulated or down-regulated in USP49 knockdown cells. Statistical significance was calculated using a Student's $t$-test. (E) Real-time RT-PCR analysis of intron 4 of RPS3 (left) and RPS6 (right) genes in control and USP49 knockdown cells. USP49 knockdown is associated with an increase in intron levels. $(F, G)$ Real-time quantitative PCR (qPCR) analysis of intron 4 of RPS3 (F) and RPS6 $(G)$ in chromatin-bound and nucleoplasmic RNA fractions. Blue bars indicate intron levels in controls. Red bars represent intron levels in USP49 knockdown cells. 
Zhang et al.

A

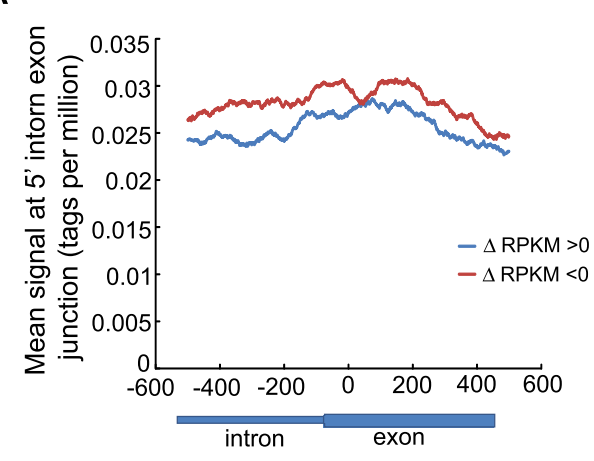

C

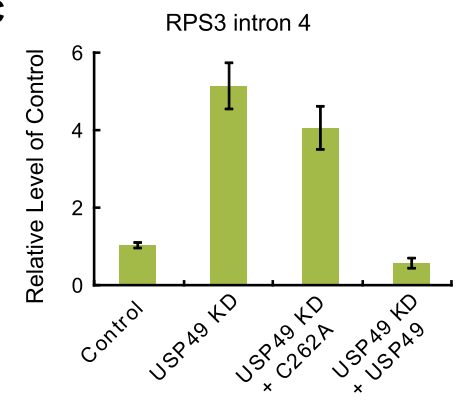

E

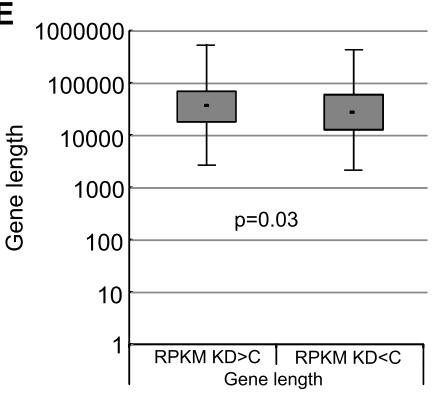

B

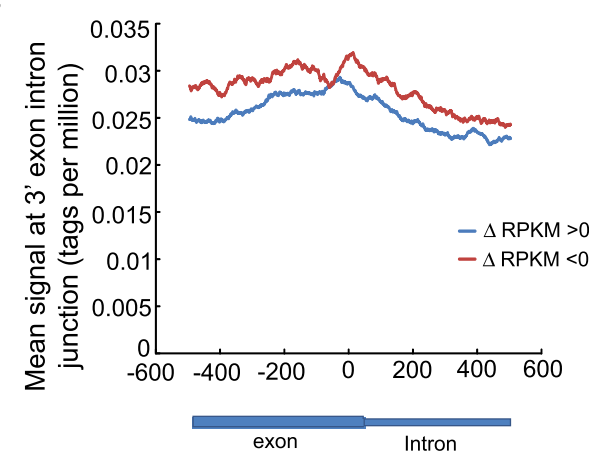

D

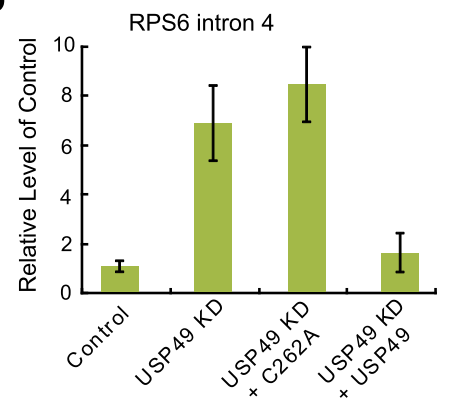

$\mathbf{F}$

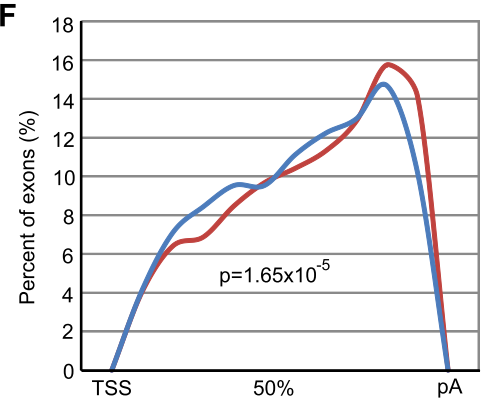

Figure 5. USP49 is enriched at a subset of down-regulated exons and regulates exon splicing. $(A, B)$ A metaexon analysis of USP49 ChIP-seq signal at the $5^{\prime}$ intron-exon boundary $(A)$ or $3^{\prime}$ exon-intron boundary $(B)$ from exons up-regulated (blue; $\left.n=4038\right)$ or downregulated (red; $n=2995$ ) in USP49 knockdown cells. Coordinates are numbered relative to the $5^{\prime}$ intron-exon or 3' exon-intron boundaries. The average number of tags that were found at each position is shown on the $Y$-axis normalized according to tags per million total unique tags. $(C, D)$ Real-time qPCR of RPS4 $(C)$ and RPS6 $(D)$ intron 4 in control or USP49 knockdown cells transfected with wild-type or C262A mutant USP49. The defects in intron splicing of RPS3 and RPS6 transcripts can be rescued by expression of wild-type but not C262A mutant USP49. (E) A box and whisker plot showing the median length of genes containing exons up-regulated or down-regulated in USP49 knockdown cells. Statistical significance was calculated using a Student's $t$-test. $(F)$ A diagram depicting the percent of exons up-regulated (blue) or down-regulated (red) at different relative positions along the gene body. Down-regulated exons are preferentially located toward the $3^{\prime}$ end of the gene. Statistical significance was calculated using a Student's $t$-test. (TSS) Transcription start site; (pA) cleavage/polyadenylation site.

genes (Supplemental Fig. S13). These results establish USP49 as a unique regulator of cotranscriptional splicing.

The efficiency of cotranscriptional splicing is known to be affected by a number of factors, including splice site strength, transcription level, and proximity to the $5^{\prime}$ and 3' ends of a gene (Ameur et al. 2011; Tilgner et al. 2012). To determine whether exons regulated by USP49 are enriched for these characteristics, we analyzed these exons. As shown in Figure 5, E and F, and Supplemental Figure S14, these exons are from shorter genes, are located closer to the transcription termination site, and have generally elevated 3 ' splice site strength. These results suggest that USP49 binding enhances the inhibitory effects of gene length and 3 ' end proximity and suppresses the stimulatory effect of $3^{\prime}$ splice strength on splicing.

\section{USP49 knockdown leads to an accumulation of uH2B at affected exons}

To determine whether the widespread defects in premRNA splicing in USP49 knockdown cells are related to changes in uH2B level, we performed anti-uH2B ChIP 
sequencing (ChIP-seq) experiments. Consistent with the roles of $\mathrm{H} 2 \mathrm{~B}$ ubiquitination in transcription, $\mathrm{uH} 2 \mathrm{~B}$ and USP49 were highly enriched in the coding regions of the genome (Fig. 6A). uH2B forms a broad peak that begins just downstream from the transcriptional start site and tapers off across the gene body before returning to basal levels near the 3 ' end (Fig. 6A, blue line). The distribution of $\mathrm{uH} 2 \mathrm{~B}$ is consistent with previously published reports (Minsky et al. 2008; Jung et al. 2012). USP49 occupancy peaks sharply at the transcriptional start site and decreases over the gene body (Fig. 6A, green line). Notably, the USP49 peak at the transcription start site is associated with a local decrease in uH2B levels (Fig. 6A, green line and blue line). This dip appears to be significant even after accounting for lower levels of nucleosomes near the transcription start site. Genes enriched for uH2B were also enriched for USP49 $\left(R^{2}=0.765502\right)$ (Supplemental Fig. S15). In USP49 knockdown cells, the levels of uH2B increased (Fig. 6A, red line), supporting our biochemical studies. Genes directly bound by USP49 show the greatest increase in uH2B levels in USP49 knockdown cells (Supplemental Fig. S16). Intriguingly, uH2B levels at exons down-regulated in USP49 knockdown cells also show a greater sensitivity to USP49 knockdown than upregulated exons (Fig. 6B). The change in uH2B levels is significantly greater at down-regulated exons as well as regions ( $\pm 500 \mathrm{nt})$ spanning their $5^{\prime}$ and $3^{\prime}$ splice sites than up-regulated exons and their splice sites (Fig. 6B). As shown in Figure 6C, USP49 binding to the regions around intron 2 and exons 2 and 3 of the SLMO2 gene, which shows defects in splicing in USP49 knockdown cells, is correlated with a significant increase in uH2B level in USP49 knockdown cells (Fig. 6C, cf. USP49 [top panel] and $\mathrm{uH} 2 \mathrm{~B}$ control and knockdown levels [bottom two panels]). More than two-thirds of exons displaying lower splicing efficiency in USP49 knockdown cells are either enriched for USP49 or enriched for UH2B after USP49 knockdown (Supplemental Fig. S17). The strong link between H2B ubiquitination, USP49 binding, and decreased splicing efficiency suggests that USP49 might regulate splicing through $\mathrm{H} 2 \mathrm{~B}$ ubiquitination. To test this hypothesis, we transfected wild-type $\mathrm{H} 2 \mathrm{~B}$ and the ubiquitination site mutant $\mathrm{H} 2 \mathrm{~B}$ K120R into control and USP49 knockdown cells and measured the effects on splicing. As shown in Figure 6, D and E, splicing defects induced by USP49 knockdown were evident in cells transfected with wild-type histone H2B (Fig. 6D,E, cf. control and USP49 knockdown); however, the effects were suppressed in cells expressing the H2B K120R mutant (Fig. 6D,E, cf. control and USP49 knockdown). Therefore, although USP49 may have other substrates, this result demonstrates that USP49 regulates pre-mRNA splicing largely through $\mathrm{H} 2 \mathrm{~B}$ deubiquitination.

Previous studies indicate that nucleosome positioning and histone modifications around the $5^{\prime}$ and $3^{\prime}$ splice sites are highly conserved and may play important roles in splice site recognition and selection (Andersson et al. 2009; Tilgner et al. 2009; Fox-Walsh and Fu 2010). Our data indicate that $\mathrm{uH} 2 \mathrm{~B}$ fold change at the $5^{\prime}$ and $3^{\prime}$ splice sites is greater for the group of exons displaying lower median levels of splicing in USP49 knockdown cells. To determine whether USP49 regulates nucleosome positioning and histone modifications at splice sites, we analyzed nucleosome density and uH2B levels across $5^{\prime}$ and $3^{\prime}$ splice sites of down-regulated and up-regulated exons. Nucleosome density and positioning, as judged by histone $\mathrm{H} 3$ abundance, are similar for the sets of downregulated and up-regulated exons (Supplemental Fig. S18), and differences in tag density in control and USP49 knockdown cells are not dramatic (Supplemental Fig. S19). Fold change in uH2B levels is significantly increased near down-regulated exons in USP49 knockdown cells, with the greatest fold change at nucleosomes positioned upstream of the $3^{\prime}$ splice site (5' end of the exon) (Fig. 6F). Intriguingly, although $\mathrm{H} 3$ levels are relatively stable (Supplemental Fig. S18), we detected a dramatic increase in $\mathrm{H} 2 \mathrm{~B}$ levels across, downstream from, and upstream of exons (Fig. 6G; Supplemental Fig. S20). Although H2B levels increase at both up-regulated and down-regulated exons in USP49 knockdown cells, the effect is most evident at down-regulated exons, which also show the greatest increase in $\mathrm{uH} 2 \mathrm{~B}$. These data are consistent with previous experiments showing that $\mathrm{uH} 2 \mathrm{~B}$ stabilizes nucleosomes in vivo (Chandrasekharan et al. 2009). These results suggest that USP49 bound at exons regulates local uH2B levels, thereby affecting nucleosome stability, a fundamental chromatin characteristic thought to play profound roles in transcription elongation and cotranscriptional splicing (Chandrasekharan et al. 2010; Dujardin et al. 2013).

\section{USP49-mediated H2B deubiquitination regulates splicing factor mRNA binding}

Splicing is a largely cotranscriptional process catalyzed by the spliceosome, whose assembly is initiated by U1 small nuclear ribonucleoprotein (snRNP) recognition of and binding to the $5^{\prime}$ splice site and U2B association with the $3^{\prime}$ branch point. The association of splicing factors with chromatin influences splicing, and histone modifications and chromatin features are thought to regulate these processes (Luco et al. 2010; Hnilicova et al. 2011; Saint-Andre et al. 2011; Schor et al. 2012; Gomez Acuna et al. 2013). To determine whether H2B ubiquitination levels at exons regulate splicing factor binding, we measured the binding of U1A and U2B to MAD2L1 nascent pre-mRNA transcripts in control and USP49 knockdown cells. RT-qPCR analysis of control and USP49 knockdown cells confirmed a significant increase of intron 2-containing transcripts in USP49 knockdown cells (Fig. 7A,C). RNA immunoprecipitation results reveal that U1A binding to the $5^{\prime}$ exon-intron junction and U2B binding to the $3^{\prime}$ intron-exon junction was dramatically decreased in USP49 knockdown cells (Fig. 7B,D, region 2). To ensure that the decreased U1A binding is not a general effect of USP49 knockdown, we determined U1A binding at the $5^{\prime}$ splice site of $\beta$-actin nascent transcripts, which are normally spliced in USP49 knockdown cells. U1A is recruited normally to the $5^{\prime}$ splice site of $\beta$-actin nascent transcripts in USP49 knockdown cells (Supplemental 


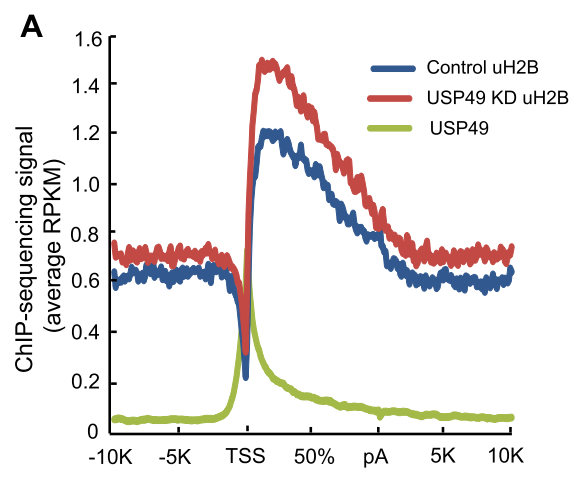

B
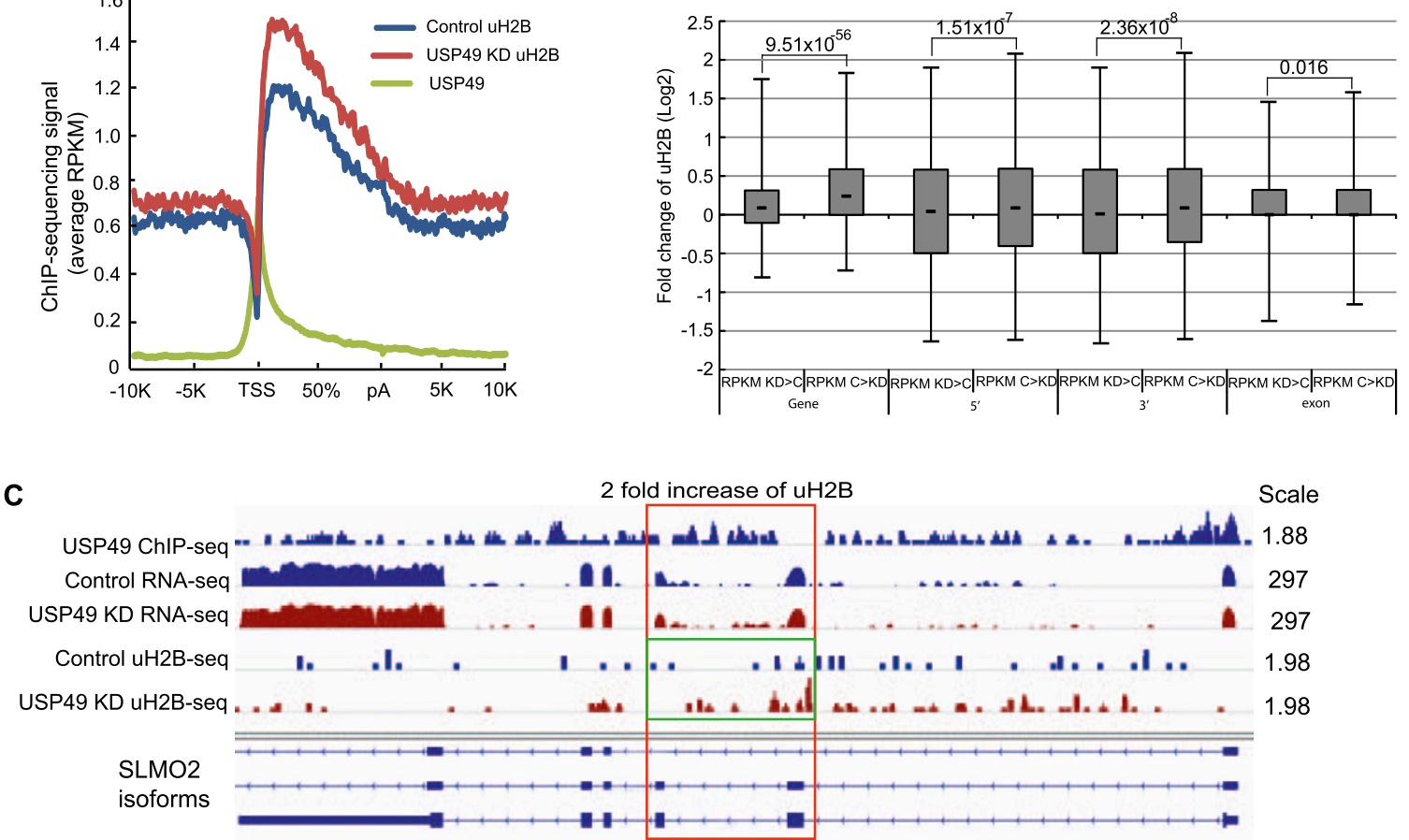

D MAD2L1 intron 2

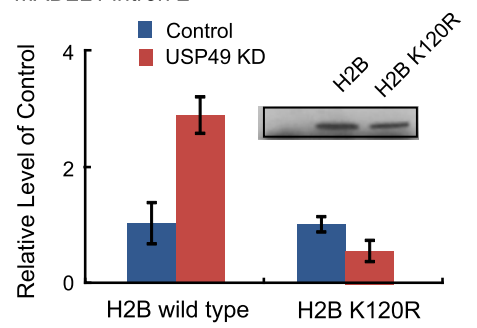

$\mathbf{F}$

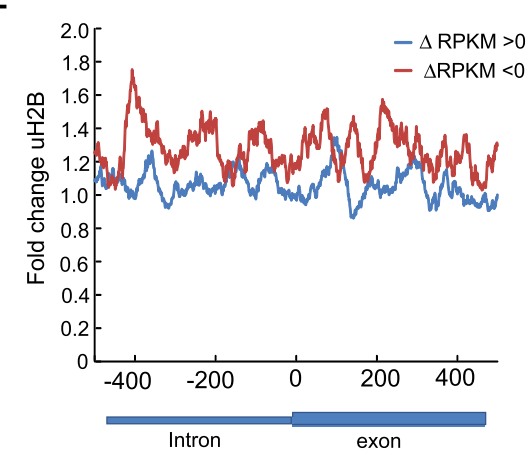

$\mathbf{E}$

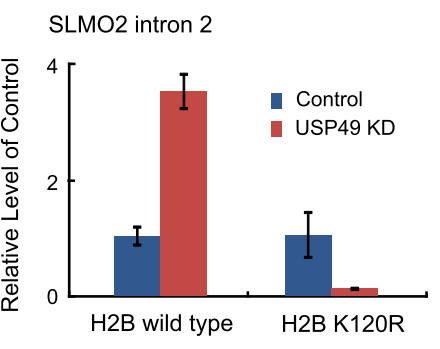

G

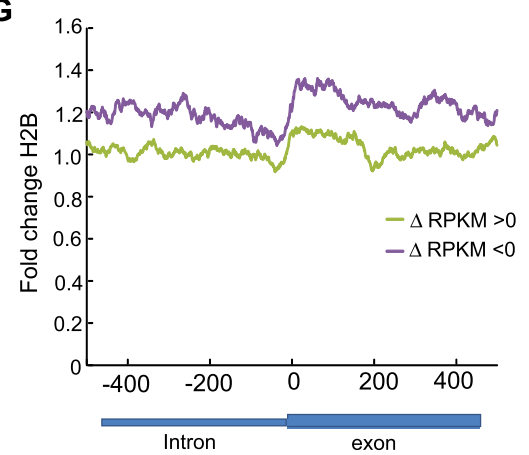

Figure 6. USP49 knockdown results in an increase of uH2B level. (A) Average USP49 (green) and uH2B (control [blue]; USP49 knockdown [red]) occupancy profiles across transcribed regions of expressed genes plus $10 \mathrm{~kb}$ upstream of and downstream from the transcriptional start and transcriptional termination sites. (TSS) Transcription start site; (pA) cleavage/polyadenylation site. (B) Fold change $\left(\log _{2}\right)$ in uH2B levels of exons up-regulated or down-regulated in USP49 knockdown cells. $\log _{2}$ fold change is significantly greater at down-regulated exons and their $3^{\prime}$ and $5^{\prime}$ splice sites. Statistical significance was calculated using a Student's $t$-test. $(C)$ A representative image of SLMO2 with aligned tags from the USP49 ChIP-seq, RNA-seq, and uH2B ChIP-seq in control and USP49 knockdown cells. Control samples are shown in blue, and USP49 knockdown samples are shown in red. The red box indicates the region with lower SC in USP49 knockdown cells. $(D, E)$ Real-time RT-PCR analysis of MAD2L1 intron $2(D)$ and SLMO2 intron $2(E)$ in control and USP49 knockdown cells transfected with wild-type H2B and the H2B K120R mutant. Transfection of wild-type H2B does not interfere with USP49 knockdown-induced splicing defects, but transfection of H2B K120R abolishes USP49 knockdown-induced splicing defects. A Western blot assay of the expression level of wild-type and K120R mutant H2B is inset in $D$. $(F, G)$ A metaexon analysis of fold change in $\mathrm{UH2B}(F)$ and $\mathrm{H} 2 \mathrm{~B}(G)$ ChIP-seq signal at the $5^{\prime}$ intron-exon boundary from exons up-regulated (blue; $n=4038$ ) or down-regulated (red; $n=2995$ ) in USP49 knockdown cells. Coordinates are numbered relative to the 5 ' intron-exon boundary. 
A

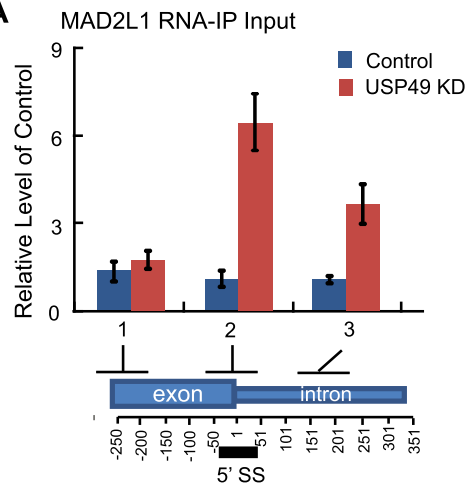

C

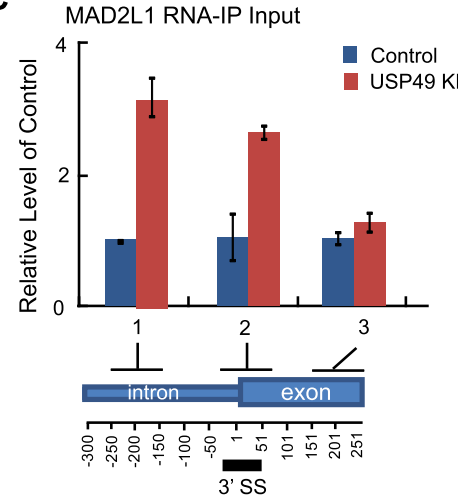

E

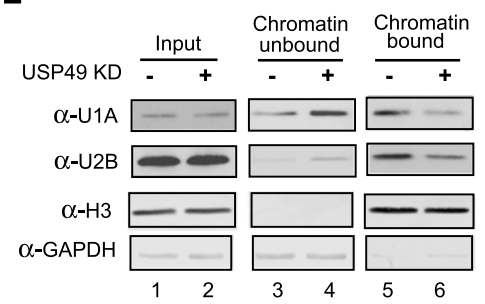

B MAD2L1 RNA-IP $(\alpha-\mathrm{U} 1 \mathrm{~A})$

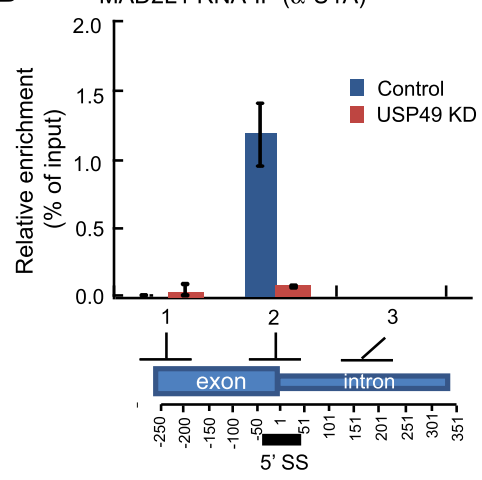

D

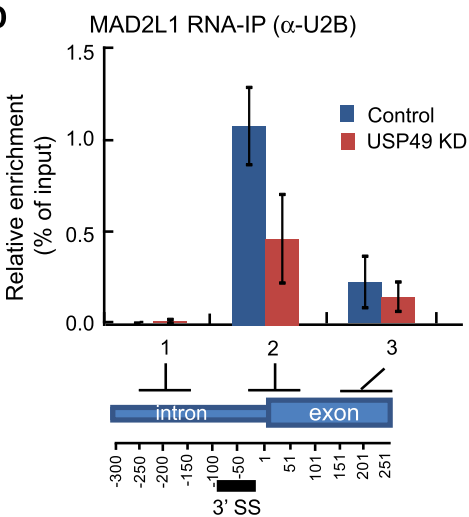

$\mathbf{F}$

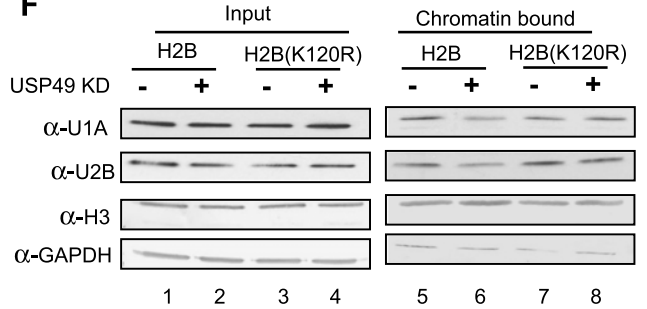

G

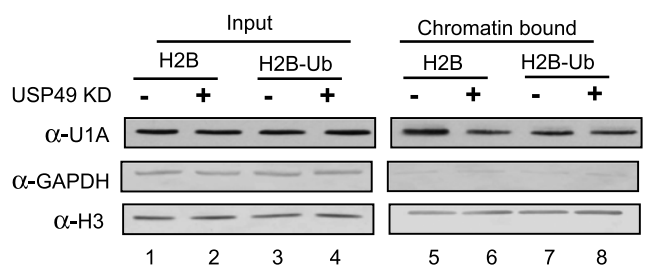

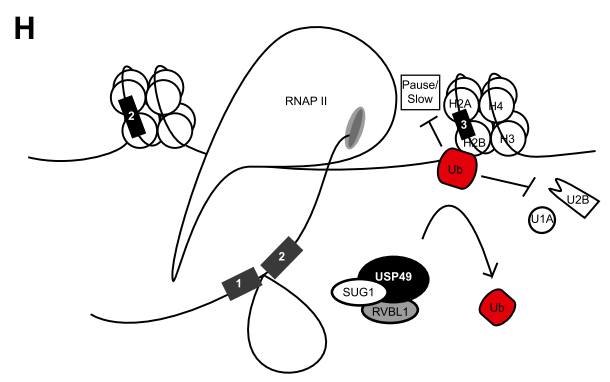

Figure 7. USP49 and H2B deubiquitination regulates U1A and U2B association with chromatin and mRNA. $(A, B)$ RT-qPCR analysis of input $(A)$ and U1A RNA immunoprecipitates $(B)$ on MAD2L1 transcript. Regions for real-time PCR amplification, corresponding to exon-intron junctions, are shown at the bottom. $(C, D)$ RT-qPCR analysis of input $(C)$ and U2B RNA immunoprecipitates $(D)$ on MAD2L1 transcript. Regions for real-time PCR amplification, corresponding to exon-intron junctions, are shown at the bottom. (E) Western blot assay of U1A and U2B binding to chromatin in control and USP49 knockdown cells. Antibodies used are labeled at the left side of the panels. (F) Western blot assay of U1A and U2B binding to chromatin in USP49 control and USP49 knockdown cells transfected with wild-type H2B and the H2B K120R mutant. Transfection of wild-type H2B has no effect on USP49 knockdown-induced decrease of U1A or U2B binding to chromatin, but transfection of H2B K120R abolishes the USP49 knockdown-induced decrease of U1A and U2B binding to chromatin. $(G)$ H2B-ubiquitin fusion protein reduces the association of U1A with chromatin. Western blot assay of U1A tethering to chromatin in control and USP49 knockdown cells transfected with H2B and H2B-ubiquitin fusion constructs. (H) A proposed model of USP49-regulated cotranscriptional splicing. USP49 regulates uH2B levels at specific exons and 3' and 5' splice regions. In USP49 knockdown cells, uH2B levels are increased at these regions. uH2B enhances nucleosome stability, altering RNAP II elongation. The RNAP II elongation rate has been shown to regulate cotranscriptional splicing efficiency and splicing factor recruitment and favor alternative splicing. 
Fig. S21). Previous studies suggest that U1A is constitutively associated with RNAP II, while U2B is conditionally associated with RNAP II (Spiluttini et al. 2010; Gu et al. 2013). To determine whether USP49 regulates the association of U1A and U2B with chromatin, we performed anti-U1A and anti-U2B ChIP. Intriguingly, we found that both chromatin-bound U1A and U2B are decreased in USP49 knockdown cells (Supplemental Fig. S22A,B). Confirming these studies, Western blot analysis of formaldehyde cross-linked cell lysates revealed that USP49 knockdown significantly reduced the tethering of $\mathrm{U} 1 \mathrm{~A}$ and $\mathrm{U} 2 \mathrm{~B}$ to chromatin and that this reduction in chromatin-tethered U1A and U2B is offset by an increase of U1A and U2B levels in nonchromatin fractions (Fig. 7E, top two panels, cf. lanes 5 and 6 and lanes 3 and 4). Total levels of U1A and U2B are similar in control and knockdown cells (Fig. 6C, top two panels, cf. lanes 1 and 2), indicating that USP49 knockdown affects U1A and U2B localization but not expression. Importantly, USP49 regulates U1A localization through $\mathrm{H} 2 \mathrm{~B}$ deubiquitination. As shown in Figure 7F, expression of the H2B K120R mutant suppresses changes in U1A and U2B localization induced by USP49 knockdown, while expression of wildtype H2B cannot (Fig. 7F, cf. lanes 5,6 and 7,8). Confirming these results, expression of a ubiquitin-H2B fusion protein phenocopies USP49 knockdown, reducing U1A and U2B association with chromatin (Fig. 7G, cf. lanes 7,8 and 5). Together, these data suggest that $\mathrm{H} 2 \mathrm{~B}$ deubiquitination, as regulated by USP49, controls splicing factor binding to specific nascent pre-mRNAs.

\section{Discussion}

In this study, we identify USP49 as a histone H2B-specific deubiquitinase (Fig. 1) and demonstrate that USP49mediated $\mathrm{H} 2 \mathrm{~B}$ deubiquitination is an important regulator of splicing (Fig. 5). Unlike previously characterized H2B deubiquitinases, which exhibit $\mathrm{uH} 2 \mathrm{~B}$ and $\mathrm{uH} 2 \mathrm{~A}$ dualsubstrate specificity, the USP49 complex specifically deubiquitinates $\mathrm{uH} 2 \mathrm{~B}$ in vitro and in vivo (Figs. 2, 3). The significant change of uH2B levels in USP49 knockdown cells suggests that USP49 is an important regulator of $\mathrm{uH} 2 \mathrm{~B}$ levels in vivo (Figs. 3, 6). In the absence of USP49, exon and splice site chromatin architecture is perturbed; increased $\mathrm{uH} 2 \mathrm{~B}$ impairs the association of $\mathrm{U} 1 \mathrm{~A}$ and $\mathrm{U} 2 \mathrm{~B}$ with chromatin and decreases binding of spliceosome components U1A and U2B to mRNA transcripts, resulting in defects in cotranscriptional splicing (Figs. 4, 6, 7). Therefore, this study identifies USP49 as a novel H2Bspecific deubiquitinase and uncovers critical roles for H2B deubiquitination in splicing.

In eukaryotic cells, gene transcription is coupled to RNA processing events such as splicing, polyadenylation, and $5^{\prime}$ capping. During transcription, RNAP II serves as a platform for assembly of both mRNA processing machinery and chromatin-modifying/remodeling complexes (for review, see Shukla and Oberdoerffer 2012). Links between chromatin and mRNA processing are supported by several recent reports indicating that exons and introns possess subtly different chromatin architecture
(Schwartz et al. 2009; Dhami et al. 2010; Wilhelm et al. 2011). Marks of active transcription, including H3K36, H3K4, and H3K79 methylations, preferentially decorate exons, while repressive marks are enriched in introns (Andersson et al. 2009; Schwartz et al. 2009; Dhami et al. 2010; Huff et al. 2010; Wilhelm et al. 2011). These modifications appear to play functional roles; both H3K36 and H3K4 methylation have been shown to direct splicing events (for review, see Luco et al. 2011). uH2B also marks exon-intron junctions in both humans and yeasts and has been implicated in spliceosome recruitment through interaction with the SR-like protein $\mathrm{Npl} 3$ in yeast (Shieh et al. 2011; Jung et al. 2012). Our data revealed that, upon USP49 knockdown, a large subset of exons is differentially and incompletely spliced (Fig. 4). Importantly, the splicing defect evident upon USP49 knockdown is rescued by expression of an ubiquitination site $\mathrm{H} 2 \mathrm{~B}$ mutant, demonstrating that USP49 regulates splicing through H2B deubiquitination (Fig. 6D,E). USP49 recruitment is increased at a subset of exons whose expression is decreased in USP49 knockdown cells (Fig. 5A,B). USP49 knockdown promotes the increase in $\mathrm{uH} 2 \mathrm{~B}$ and $\mathrm{H} 2 \mathrm{~B}$ levels at these exons and regions flanking the upstream $3^{\prime}$ and downstream $5^{\prime}$ splice sites, inhibiting splicing factor association with RNAP II and spliceosome assembly on nascent RNA (Fig. 7). Therefore, this study provides direct evidence linking $\mathrm{H} 2 \mathrm{~B}$ deubiquitination and pre-mRNA splicing.

How chromatin modifications may inform splicing decisions is an area of active research. Previous studies linking histone methylation and acetylation to splice site choice suggest that chromatin-spliceosome adaptor proteins may mediate these regulations (Luco et al. 2010; Hnilicova et al. 2011; Saint-Andre et al. 2011; Schor et al. 2012). Chromatin modifications may also regulate splicing by modulating the RNAP II elongation rate, a potent regulator of both alternative splicing and cotranscriptional splicing (for review, see Luco et al. 2011; Gomez Acuna et al. 2013). Our data suggest a heretofore unknown role for $\mathrm{H} 2 \mathrm{~B}$ deubiquitination in splicing factor localization and mRNA binding, linking this chromatin modification with the core splicing machinery (Fig. 7). Splicing is initiated upon binding of the U1 snRNP to the 5 ' splice site and of the U2 snRNP to the branch point. We found that recruitment of U1A and $\mathrm{U} 2 \mathrm{~B}$, core components of the U1 and U2 snRNPs, is severely diminished at the $5^{\prime}$ and 3 ' splice junctions of affected but not unaffected exons in USP49 knockdown cells (Fig. 7; Supplemental Fig. S21). The impaired U1A and U2B binding typical of USP49 knockdown cells can be mimicked by expression of a ubiquitin-H2B fusion protein (Fig. 7G). How ubiquitinated $\mathrm{H} 2 \mathrm{~B}$ affects $\mathrm{U} 1 \mathrm{~A}$ and $\mathrm{U} 2 \mathrm{~B}$ exclusion is not yet clear, but the known roles of $\mathrm{uH} 2 \mathrm{~B}$ suggest several possibilities. Ubiquitinated $\mathrm{H} 2 \mathrm{~B}$ causes local changes in chromatin structure, as evidenced by the increase in $\mathrm{H} 2 \mathrm{~B}$ relative to $\mathrm{H} 3$ seen in our data (Fig. 6G; Supplemental Fig. S18). Chromatin remodeling factors facilitate RNAP II elongation by partially disassembling nucleosomes ahead of RNAP II (Kulaeva et al. 2013). This involves the disassociation of a $\mathrm{H} 2 \mathrm{~B} / \mathrm{H} 2 \mathrm{~A}$ dimer, while the $\mathrm{H} 3 / \mathrm{H} 4$ 
tetramer is thought to remain associated with chromatin (Kireeva et al. 2002). In USP49 knockdown cells, uH2B is increased at down-regulated exons, enhancing the stability of the nucleosome spanning these exons. These stable nucleosomes could alter RNAP II elongation rates, thereby lowering transcription level, increasing alternative splicing, and decreasing cotranscriptional splicing of these $\mathrm{uH} 2 \mathrm{~B}$-containing nucleosome-enriched exons (Fig. 7H; Carrillo Oesterreich et al. 2011; Shukla and Oberdoerffer 2012). In addition, uH2B is also an upstream regulator of histone H3 Lys 4 and Lys 36 methylation, modifications known to regulate localization of the splicing machinery (Zhang 2003; Luco et al. 2011). Our data suggest a model in which uH2B levels at exons and exonadjacent regions modulate splicing factor localization and binding and that USP49 regulates the utilization of these splice sites through H2B deubiquitination. Our studies expand the roles of chromatin in splicing beyond alternative exon inclusion and suggest that chromatin features are significant regulators of constitutive splicing events.

\section{Materials and methods}

\section{Constructs, cell lines, and antibodies}

Full-length cDNA of USP49 was purchased from Open Biosystems (MHS1011-76095) and cloned into the pcDNA3-Flag vector, pFastBacHTb vector (Invitrogen), and retroviral pMIGR1 vector (Joo et al. 2011) through PCR and verified by sequencing. C262A point mutation was carried out through PCR-directed mutagenesis and verified by sequencing. Recombinant baculoviruses were generated and amplified following the manufacturer's protocol (Joo et al. 2011). Since GFP and Flag-HA-USP49 were produced in the same bicistronic transcript, cell sorting for GFP expression was done twice to enrich GFP- and USP49positive cells to $>90 \%$ of cells.

Human adenocarcinomic alveolar basal epithelial cell line A549 and SV40 T antigen transformed human embryonic kidney 293 T cells were purchased from American Type Culture Collection. A549 cells were cultured in DMEM/F12 (Invitrogen) supplemented with $10 \%$ FBS (Hyclone) and $1 \%$ ampicillin-streptomycin (Hyclone). 293T cells were cultured in DMEM (Hyclone) supplemented with $10 \%$ FBS (Hyclone) and $1 \%$ ampicillin-streptomycin (Hyclone). Tetracycline-inducible USP49 knockdown HCT116 cells were established using the T-REx expression system (Invitrogen). Briefly, HCT116 cells were transfected with pcDNA6/ TR. The stable clones were selected and maintained by blasticidin.

To knock down USP49, two different shRNA oligonucleotides (sense strands: GGTCATGCATCACGGGAAA and GGACTAC GTGCTCAATGAT) were synthesized (Integrated DNA Technologies), cloned into plasmid pBabe-U6 (kindly provided by Dr. Xinbin Chen, University of California at Davis), and used to transfect pcDNA6/TR-positive colonies (Yan et al. 2008). The transfected cells were then cultured with puromycin, and the positive clones were screened for tetracycline-inducible knockdown of USP49 by Western blot assay.

USP49 antibody was generated by standard protocols and affinity-purified by antigen column. Anti-ubiquitin-H2B antibody was kindly provided by Dr. Moshe Oren (Weizmann Institute of Science, Israel) and was also purchased from Millipore (catalog no. 05-1312). Antibody against ubiquitinated H2A (catalog no. 05-678) was purchased from Millipore. Anti-Flag antibody (catalog no. F3165) and anti-Flag M2 affinity gel (catalog no. A2220) were purchased from Sigma. Antibodies against RVB1 (catalog no. ab51500) and SUG1 (catalog no. ab3323) were purchased from Abcam. The anti-HA antibody was derived from the mouse hybridoma 12CA5.

\section{In vitro and in vivo histone deubiquitination assay}

uH2A-containing nucleosomes were isolated from HeLa stable cell lines expressing Flag-H2A and HA-ubiquitin, and uH2Acontaining core histones were prepared from these nucleosomes by hydroxyapatite columns (Joo et al. 2007, 2011). uH2B-containing nucleosomes were purified from a yeast strain expressing Flaghuman H2B by anti-Flag M2 affinity purification, and core histones were obtained by hydroxyapatite columns (Joo et al. 2011). Histone deubiquitination reactions were performed as described (Joo et al. 2007). For in vivo histone deubiquitination assays, control or siRNA oligonucleotides against USP49 purchased from Invitrogen in a purified, annealed duplex form were transfected into cells using Lipofectamine 2000 (Wang et al. 2006; Wei et al. 2006). USP49 and USP49 C262A mutants were transfected into cells with Effectene following the manufacturer's instructions. A Western blot assay with anti-uH2A and anti-uH2B antibodies on transfected cells was performed as described (Joo et al. 2007).

\section{ChIP-seq and RNA-seq}

mRNA samples were prepared as described in the Illumina mRNA sequencing sample preparation guide. ChIP was prepared following a protocol provided by Dr. Zhibin Wang (Johns Hopkins University) (Wang et al. 2009). Reads were aligned with TopHat (RNA-seq) or Bowtie (ChIP-seq) in the University of Alabama at Birmingham (UAB) instance of Galaxy. Additional analyses were performed in BedTools, Samtools, or PICARD or using custom scripts. Statistical analyses were performed in R.

\section{Acknowledgments}

We thank Dr. Moshe Oren for sending the anti-uH2B antibody prior to its commercialization and the wild-type and mutant (K120R and K125R) H2B plasmids, Dr. Steven B. McMahon for USP22 shRNA, Dr. Xinbin Chen for HCT116 cells with pcDNA6TR transfection and pBabe-U6 plasmid, Dr. Kenshi Komatsu for wild-type H2B and H2B K120R mutant plasmids, Dr. Tsukasa Shimojima for the initial cloning of USP49, Ms. Elizabeth Chang for help with sample preparation for mass spectrometry, and the University of Alabama at Birmingham Fermentation Facility for mammalian cell culture. H.W. is a Leukemia and Lymphoma Scholar and is supported by NIH grant (GM081489). L.M. is supported by grants from the National Basic Research Program of China (973 Program, 2012CB910900 and 2012CB1 14200). H.E.-B. and P.T. are supported by National Cancer Institute Cancer Center support grant P30 CA08748 to the Microchemistry and Proteomics Core Laboratory. Z.Z. is partially supported by the University of Alabama at Birmingham Comprehensive Cancer Center Junior Faculty Development Grant Program (CA13148-39). A.J. is partially supported by the Carmichael Fund.

\section{References}

Allo M, Buggiano V, Fededa JP, Petrillo E, Schor I, de la Mata M, Agirre E, Plass M, Eyras E, Elela SA, et al. 2009. Control of alternative splicing through siRNA-mediated transcriptional gene silencing. Nat Struct Mol Biol 16: 717-724.

Ameur A, Zaghlool A, Halvardson J, Wetterbom A, Gyllensten U, Cavelier L, Feuk L. 2011. Total RNA sequencing reveals 
nascent transcription and widespread co-transcriptional splicing in the human brain. Nat Struct Mol Biol 18: 14351440.

Andersson R, Enroth S, Rada-Iglesias A, Wadelius C, Komorowski J. 2009. Nucleosomes are well positioned in exons and carry characteristic histone modifications. Genome Res 19: 17321741.

Batta K, Zhang Z, Yen K, Goffman DB, Pugh BF. 2011. Genomewide function of $\mathrm{H} 2 \mathrm{~B}$ ubiquitylation in promoter and genic regions. Genes Dev 25: 2254-2265.

Buszczak M, Paterno S, Spradling AC. 2009. Drosophila stem cells share a common requirement for the histone $\mathrm{H} 2 \mathrm{~B}$ ubiquitin protease Scrawny. Science 323: 248-251.

Carrillo Oesterreich F, Bieberstein N, Neugebauer KM. 2011. Pause locally, splice globally. Trends Cell Biol 21: 328-335.

Chandrasekharan MB, Huang F, Sun ZW. 2009. Ubiquitination of histone $\mathrm{H} 2 \mathrm{~B}$ regulates chromatin dynamics by enhancing nucleosome stability. Proc Natl Acad Sci 106: 16686-16691.

Chandrasekharan MB, Huang F, Sun ZW. 2010. Histone H2B ubiquitination and beyond: Regulation of nucleosome stability, chromatin dynamics and the trans-histone $\mathrm{H} 3$ methylation. Epigenetics 5: 460-468.

Daniel JA, Torok MS, Sun ZW, Schieltz D, Allis CD, Yates JR III, Grant PA. 2004. Deubiquitination of histone H2B by a yeast acetyltransferase complex regulates transcription. I Biol Chem 279: 1867-1871.

Dhami P, Saffrey P, Bruce AW, Dillon SC, Chiang K, Bonhoure N, Koch CM, Bye J, James K, Foad NS, et al. 2010. Complex exon-intron marking by histone modifications is not determined solely by nucleosome distribution. PLOS ONE 5: e12339.

Dujardin G, Lafaille C, Petrillo E, Buggiano V, Gomez Acuna LI, Fiszbein A, Godoy Herz MA, Nieto Moreno N, Munoz MJ, Allo M, et al. 2013. Transcriptional elongation and alternative splicing. Biochim Biophys Acta 1829: 134-140.

Emre NCT, Ingvarsdottir K, Wyce A, Wood A, Krogan NJ, Henry KW, Li K, Marmorstein R, Greenblatt JF, Shilatifard A, et al. 2005. Maintenance of low histone ubiquitylation by Ubp10 correlates with telomere-proximal Sir2 association and gene silencing. Mol Cell 17: 585-594.

Ezhkova E, Tansey WP. 2004. Proteasomal ATPases link ubiquitylation of histone H2B to methylation of histone H3. Mol Cell 13: 435-442.

Fleming AB, Kao CF, Hillyer C, Pikaart M, Osley MA. 2008. H2B ubiquitylation plays a role in nucleosome dynamics during transcription elongation. Mol Cell 31: 57-66.

Fox-Walsh K, Fu XD. 2010. Chromatin: The final frontier in splicing regulation? Dev Cell 18: 336-338.

Fuchs G, Shema E, Vesterman R, Kotler E, Wolchinsky Z, Wilder S, Golomb L, Pribluda A, Zhang F, Haj-Yahya M, et al. 2012. RNF20 and USP44 regulate stem cell differentiation by modulating H2B monoubiquitylation. Mol Cell 46: 662-673.

Gardner RG, Nelson ZW, Gottschling DE. 2005. Ubp10/Dot4p regulates the persistence of ubiquitinated histone $\mathrm{H} 2 \mathrm{~B}$ : Distinct roles in telomeric silencing and general chromatin. Mol Cell Biol 25: 6123-6139.

Gomez Acuna LI, Fiszbein A, Allo M, Schor IE, Kornblihtt AR. 2013. Connections between chromatin signatures and splicing. Wiley Interdiscip Rev RNA 4: 77-91.

Gu B, Eick D, Bensaude O. 2013. CTD serine-2 plays a critical role in splicing and termination factor recruitment to RNA polymerase II in vivo. Nucleic Acids Res 41: 1591-1603.

Henry KW, Wyce A, Lo WS, Duggan LJ, Emre NC, Kao CF, Pillus L, Shilatifard A, Osley MA, Berger SL. 2003. Transcriptional activation via sequential histone $\mathrm{H} 2 \mathrm{~B}$ ubiquitylation and deubiquitylation, mediated by SAGA-associated Ubp8. Genes Dev 17: 2648-2663.

Hnilicova J, Hozeifi S, Duskova E, Icha J, Tomankova T, Stanek D. 2011. Histone deacetylase activity modulates alternative splicing. PLOS ONE 6: e16727.

Huff JT, Plocik AM, Guthrie C, Yamamoto KR. 2010. Reciprocal intronic and exonic histone modification regions in humans. Nat Struct Mol Biol 17: 1495-1499.

Jenuwein T, Allis CD. 2001. Translating the histone code. Science 293: 1074-1080.

Jha S, Dutta A. 2009. RVB1/RVB2: Running rings around molecular biology. Mol Cell 34: 521-533.

Joo HY, Zhai L, Yang C, Nie S, Erdjument-Bromage H, Tempst P, Chang C, Wang H. 2007. Regulation of cell cycle progression and gene expression by $\mathrm{H} 2 \mathrm{~A}$ deubiquitination. Nature 449: 1068-1072.

Joo HY, Jones A, Yang C, Zhai L, Smith ADt, Zhang Z, Chandrasekharan MB, Sun ZW, Renfrow MB, Wang $Y$, et al. 2011. Regulation of histone H2A and H2B deubiquitination and Xenopus development by USP12 and USP46. I Biol Chem 286: 7190-7201.

Jung I, Kim SK, Kim M, Han YM, Kim YS, Kim D, Lee D. 2012. $\mathrm{H} 2 \mathrm{~B}$ monoubiquitylation is a $5^{\prime}$-enriched active transcription mark and correlates with exon-intron structure in human cells. Genome Res 22: 1026-1035.

Kireeva ML, Walter W, Tchernajenko V, Bondarenko V, Kashlev M, Studitsky VM. 2002. Nucleosome remodeling induced by RNA polymerase II: Loss of the $\mathrm{H} 2 \mathrm{~A} / \mathrm{H} 2 \mathrm{~B}$ dimer during transcription. Mol Cell 9: 541-552.

Kouzarides T. 2007. Chromatin modifications and their function. Cell 128: 693-705.

Kulaeva OI, Hsieh FK, Chang HW, Luse DS, Studitsky VM. 2013. Mechanism of transcription through a nucleosome by RNA polymerase II. Biochim Biophys Acta 1829: 76-83.

Luco RF, Pan Q, Tominaga K, Blencowe BJ, Pereira-Smith OM, Misteli T. 2010. Regulation of alternative splicing by histone modifications. Science 327: 996-1000.

Luco RF, Allo M, Schor IE, Kornblihtt AR, Misteli T. 2011. Epigenetics in alternative pre-mRNA splicing. Cell 144: 16-26.

Minsky N, Shema E, Field Y, Schuster M, Segal E, Oren M. 2008. Monoubiquitinated $\mathrm{H} 2 \mathrm{~B}$ is associated with the transcribed region of highly expressed genes in human cells. Nat Cell Biol 10: 483-488.

Moehle EA, Ryan CJ, Krogan NJ, Kress TL, Guthrie C. 2012. The yeast SR-like protein Npl3 links chromatin modification to mRNA processing. PLoS Genet 8: e1003101.

Nicassio F, Corrado N, Vissers JH, Areces LB, Bergink S, Marteijn JA, Geverts B, Houtsmuller AB, Vermeulen W, Di Fiore PP, et al. 2007. Human USP3 is a chromatin modifier required for $\mathrm{S}$ phase progression and genome stability. Curr Biol 17: 1972-1977.

Saint-Andre V, Batsche E, Rachez C, Muchardt C. 2011. Histone $\mathrm{H} 3$ lysine 9 trimethylation and $\mathrm{HP} 1 \gamma$ favor inclusion of alternative exons. Nat Struct Mol Biol 18: 337-344.

Schor IE, Rascovan N, Pelisch F, Allo M, Kornblihtt AR. 2009. Neuronal cell depolarization induces intragenic chromatin modifications affecting NCAM alternative splicing. Proc Natl Acad Sci 106: 4325-4330.

Schor IE, Lleres D, Risso GJ, Pawellek A, Ule J, Lamond AI, Kornblihtt AR. 2012. Perturbation of chromatin structure globally affects localization and recruitment of splicing factors. PLOS ONE 7: e48084.

Schulze JM, Hentrich T, Nakanishi S, Gupta A, Emberly E, Shilatifard A, Kobor MS. 2011. Splitting the task: Ubp8 and Ubp10 deubiquitinate different cellular pools of H2BK123. Genes Dev 25: 2242-2247. 
Schwartz S, Meshorer E, Ast G. 2009. Chromatin organization marks exon-intron structure. Nat Struct Mol Biol 16: 990995.

Shieh GS, Pan $\mathrm{CH}, \mathrm{Wu} \mathrm{JH}$, Sun YJ, Wang CC, Hsiao WC, Lin $\mathrm{CY}$, Tung L, Chang TH, Fleming AB, et al. 2011. H2B ubiquitylation is part of chromatin architecture that marks exon-intron structure in budding yeast. BMC Genomics 12: 627.

Shukla S, Oberdoerffer S. 2012. Co-transcriptional regulation of alternative pre-mRNA splicing. Biochim Biophys Acta 1819: 673-683.

Spiluttini B, Gu B, Belagal P, Smirnova AS, Nguyen VT, Hebert C, Schmidt U, Bertrand E, Darzacq X, Bensaude O. 2010. Splicing-independent recruitment of U1 snRNP to a transcription unit in living cells. J Cell Sci 123: 2085-2093.

Tilgner H, Nikolaou C, Althammer S, Sammeth M, Beato M, Valcarcel J, Guigo R. 2009. Nucleosome positioning as a determinant of exon recognition. Nat Struct Mol Biol 16: 9961001.

Tilgner H, Knowles DG, Johnson R, Davis CA, Chakrabortty S, Djebali S, Curado J, Snyder M, Gingeras TR, Guigo R. 2012. Deep sequencing of subcellular RNA fractions shows splicing to be predominantly co-transcriptional in the human genome but inefficient for lncRNAs. Genome Res 22: 16161625.

van der Knaap JA, Kumar BR, Moshkin YM, Langenberg K, Krijgsveld J, Heck AJ, Karch F, Verrijzer CP. 2005. GMP synthetase stimulates histone $\mathrm{H} 2 \mathrm{~B}$ deubiquitylation by the epigenetic silencer USP7. Mol Cell 17: 695-707.

Wang $\mathrm{H}$, Zhai L, Xu J, Joo H-Y, Jackson S, Erdjument-Bromage H, Tempst P, Xiong Y, Zhang Y. 2006. Histone H3 and H4 ubiquitylation by the CUL4-DDB-ROC1 ubiquitin ligase facilitates cellular response to DNA damage. Mol Cell 22: 383-394.

Wang Z, Zang C, Cui K, Schones DE, Barski A, Peng W, Zhao K. 2009. Genome-wide mapping of HATs and HDACs reveals distinct functions in active and inactive genes. Cell 138: 1019-1031.

Weake VM, Workman JL. 2008. Histone ubiquitination: Triggering gene activity. Mol Cell 29: 653-663.

Weake VM, Lee KK, Guelman S, Lin C-H, Seidel C, Abmayr SM, Workman JL. 2008. SAGA-mediated H2B deubiquitination controls the development of neuronal connectivity in the Drosophila visual system. EMBO J 27: 394-405.

Wei J, Zhai L, Xu J, Wang H. 2006. Role of Bmil in H2A ubiquitylation and Hox gene silencing. I Biol Chem 281: 22537-22544.

Wilhelm BT, Marguerat S, Aligianni S, Codlin S, Watt S, Bahler J. 2011. Differential patterns of intronic and exonic DNA regions with respect to RNA polymerase II occupancy, nucleosome density and H3K36me3 marking in fission yeast. Genome Biol 12: R82.

Wyce A, Xiao T, Whelan KA, Kosman C, Walter W, Eick D, Hughes TR, Krogan NJ, Strahl BD, Berger SL. 2007. H2B ubiquitylation acts as a barrier to Ctk1 nucleosomal recruitment prior to removal by Ubp8 within a SAGA-related complex. Mol Cell 27: 275-288.

Yan W, Liu G, Scoumanne A, Chen X. 2008. Suppression of inhibitor of differentiation 2, a target of mutant p53, is required for gain-of-function mutations. Cancer Res 68: 6789-6796.

Zhang Y. 2003. Transcriptional regulation by histone ubiquitination and deubiquitination. Genes Dev 17: 2733-2740.

Zhang XY, Varthi M, Sykes SM, Phillips C, Warzecha C, Zhu W, Wyce A, Thorne AW, Berger SL, McMahon SB. 2008. The putative cancer stem cell marker USP22 is a subunit of the human SAGA complex required for activated transcription and cell-cycle progression. Mol Cell 29: 102-111.

Zhao Y, Lang G, Ito S, Bonnet J, Metzger E, Sawatsubashi S, Suzuki E, Guezennec XL, Stunnenberg HG, Krasnov A, et al. 2008. TFTC/STAGA module mediates histone $\mathrm{H} 2 \mathrm{~A}$ and $\mathrm{H} 2 \mathrm{~B}$ deubiquitination, coactivates nuclear receptors, and counteracts heterochromatin silencing. Mol Cell 29: 92-101. 


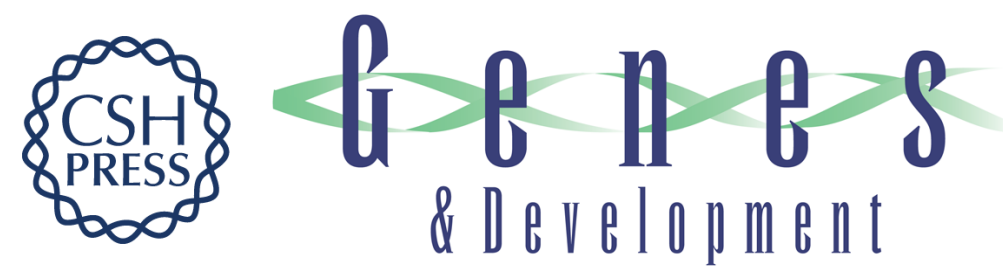

\section{USP49 deubiquitinates histone H2B and regulates cotranscriptional pre-mRNA splicing}

Zhuo Zhang, Amanda Jones, Heui-Yun Joo, et al.

Genes Dev. 2013, 27: originally published online July 3, 2013

Access the most recent version at doi:10.1101/gad.211037.112

\section{Supplemental http://genesdev.cshlp.org/content/suppl/2013/06/27/gad.211037.112.DC1 Material}

References This article cites 57 articles, 18 of which can be accessed free at: http://genesdev.cshlp.org/content/27/14/1581.full.html\#ref-list-1

\section{License}

Email Alerting

Service
Receive free email alerts when new articles cite this article - sign up in the box at the top right corner of the article or click here.

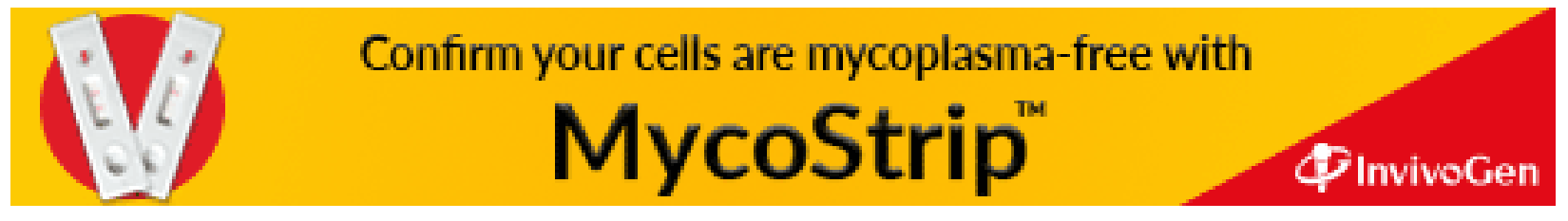

